\title{
Qualification conditions-free characterizations of the $\varepsilon$-subdifferential of convex integral functions
}

\author{
Rafael Correa, Abderrahim Hantoute, Pedro \\ Pérez-Aros
}

Received: date / Accepted: date

\begin{abstract}
We provide formulae for the $\varepsilon$-subdifferential of the integral function $I_{f}(x):=\int_{T} f(t, x) d \mu(t)$, where the integrand $f: T \times X \rightarrow \overline{\mathbb{R}}$ is measurable in $(t, x)$ and convex in $x$. The state variable lies in a locally convex space, possibly non-separable, while $T$ is given a structure of a nonnegative complete $\sigma$-finite measure space $(T, \mathcal{A}, \mu)$. The resulting characterizations are given in terms of the $\varepsilon$-subdifferential of the data functions involved in the integrand, $f$, without requiring any qualification conditions. We also derive new formulas when some usual continuity-type conditions are in force. These results are new even for the finite sum of convex functions and for the finite-dimensional setting.
\end{abstract}

Keywords Normal integrands, convex integral functionals, conjugate functions, subdifferential and $\varepsilon$-subdifferential.

\section{Introduction}

Several problems in applied mathematics such as calculus of variations, optimal control theory and stochastic programming, among others, rely on the study of integral functions and functionals given by the following expression

$$
x(\cdot) \in \mathfrak{X} \rightarrow \hat{I}_{f}(x(\cdot)):=\int_{T} f(t, x(t)) d \mu(t),
$$

This work is partially supported by CONICYT grants: Fondecyt projects N 1151003, 1190012, 1190110, and 1150909, Proyecto/Grant PIA AFB-170001, CONICYT-PCHA/doctorado Nacional/2014-21140621.

Rafael Correa, Universidad de O'Higgins and DIM-CMM of Universidad de Chile E-mail: rcorrea@dim.uchile.cl

Abderrahim Hantoute, Center for Mathematical Modeling

Universidad de Chile

E-mail: ahantoute@dim.uchile.cl

Pedro Pérez-Aros, Instituto de Ciencias de la Ingeniería

Universidad de O'higgins

E-mail: pedro.perez@uoh.com 
for a functional space $\mathfrak{X}$ and an integrand function $f$ that are given by the data of the underlying system. Problems which consider this class of functionals represent a tremendous territory for developing variational analysis, and indeed it is especially under this class of problems where the theory has traditionally been organized. Models which consider integrals with respect to time are common in the study of dynamical systems and problems of optimal control. Also, when the problem involves uncertainty, as in stochastic programming problems, the design of such mathematical models is represented by a probability space, so that the problem is modeled using the integral expression. Applications to stochastic programming problems often concern the study of density distributions, which can also be presented under integration with respect to the Lebesgue measure.

In classical studies, such as in the calculus of variations, the integrand $f(t, x)$ is usually supposed to be continuous in $t$ and $x$, jointly, or even with some order of differentiability. Later, integrands with finite values and satisfying the Carathéodory condition, that is, continuity in $x$ and measurability in $t$, have been considered. It can be easily noticed in all of these cases that for every measurable function $x(\cdot)$, the function $t \rightarrow f(t, x(t))$ is at least measurable and, hence, (1) can be well-defined using the convention adopted for the extended-real line. However, new mathematical models, especially the emergence of the modern control theory, lead to the consideration of general integrands with possibly infinite values. In this way, important kinds of constraints can most efficiently be represented. Such integrands require a distinctly new theoretical approach, where questions of measurability, meaning of the integral and the existence of measurable selections are prominent and are reflected in the concept of normal integrands.

As is traditional in optimization and generally in variational analysis, one could replace the continuity of $f_{t}:=f(t, \cdot)$ with the weaker property of lower semicontinuity, but maintaining the measurability of $f$ with respect to $t$. Nevertheless, this is not enough to ensure the measurability of $t \rightarrow f(t, x(t))$ for any measurable function $x(\cdot)$. As an example, put $T=[0,1]$ and let $\mathcal{A}$ be the $\sigma$-Algebra of Lebesgue measurable sets in $[0,1]$. If $D$ is a non-measurable set in $[0,1]$, and

$$
f(t, x):=0 \text { if } t=x \in D \text {, and } f(t, x):=1 \text { otherwise, }
$$

then the measurability and the lower semicontinuity of $f$ hold trivially. However, for $x(t)=t$ we lack the measurability of the function $t \rightarrow f(t, x(t))$. This example shows that although the lower semicontinuity assumption in $x$ is certainly right, the assumption of measurability in $t$ for each fixed $x$ is not adequate. The way out of this impasse was found by Rockafellar [32, using the concept of normal convex integrands, which is an equivalent definition to the one presented in Section 3 (see, e.g., [35, Proposition 2D], or [37, Proposition 14.39]). Such integrands are such that $f(t, \cdot)$ is proper and lower semi-continuous for each $t$, and there exists a countable collection $U$ of measurable functions $u$ from $T$ to $\mathbb{R}^{n}$ with the following properties: (a) for each $u \in U, f(t, u(t))$ is measurable in $t$; (b) for each $t, U_{t} \cap \operatorname{dom} f_{t}$ is dense in $\operatorname{dom} f_{t}$, where $U_{t}=\{u(t): u \in U\}$.

The notion of convex normal integrands provides the link that allows the connection of measurable multifunctions and the subdifferential theory. The preservation of the measurability of multifunctions under a broad variety of operations, including countable intersections and unions, sums, Painlevé-Kuratowski limits, and so on, as well as the validity of Castaing's representation theorem ( 9,10$])$, 
have made this theory very popular in various problems of applied mathematics. Castaing's representation theorem is intrinsically related to the possibility of extending the definition of the classical integration to the one of set-valued mappings, using measurable and integrable selections. In our case, we shall deal with the subdifferential mapping to get similar results as in the Leibniz integral rule.

Some of the classical results on integral functions and functionals can be found in Castaing-Valadier [10, Ioffe-Levin [23, Ioffe-Tikhomirov [24, Levin [26] and Rockafellar [32 34]. Other recent works are Borwein-Yao [5], Ioffe [22], LopezThibault [27], and Mordukhovich-Sagara [28] among others. A summary of the theory of measurability and integral functionals in finite-dimensions can be found in [35, 37, and in [10,21, 40, 41, for infinite-dimensional spaces.

The aim of this research is to give formulae for the $\varepsilon$-subdifferential of the convex integral function $I_{f}$, given by

$$
x \in X \rightarrow I_{f}(x):=\int_{T} f(t, x) d \mu(t),
$$

that is, when the space $\mathfrak{X}$ in (1) is the space of constant functions. This particular case is also known as the continuous sum. A well-known formula, given under certain continuity assumptions by Ioffe-Levin [23] for the finite-dimensional setting, establishes that

$$
\partial I_{f}(x)=\int_{T} \partial f_{t}(x) d \mu(t)+N_{\operatorname{dom} I_{f}}(x), \text { for all } x \in X,
$$

where the set $\int_{T} \partial f_{t}(x) d \mu(t)$ is understood in the sense of Aumman's integral (see Definition [5). One can compare (3) with its discrete (finite) counterpart, which states that for every two proper convex functions $f_{1}$ and $f_{2}$ such that $f_{1}$ is continuous at some point of $\operatorname{dom} f_{1} \cap \operatorname{dom} f_{2}$ one has

$$
\partial\left(f_{1}+f_{2}\right)(x)=\partial f_{1}(x)+\partial f_{2}(x) .
$$

However, in the absence of continuity assumptions, Hiriart-Urruty and Phelps formula ensures that (see, e.g., [19])

$$
\partial\left(f_{1}+f_{2}\right)(x)=\bigcap_{\varepsilon>0} \operatorname{cl}\left(\partial_{\varepsilon} f_{1}(x)+\partial_{\varepsilon} f_{2}(x)\right),
$$

provided that $f_{1}$ and $f_{2}$ are lower semi-continuous proper convex functions. At this step, a natural question is to give similar formulae for the subdifferential of integral functions as the last one above. It feels natural to think about a generalization of (3) in the following form,

$$
\partial I_{f}(x)=\bigcap_{\eta>0} \operatorname{cl}\left\{\int_{T} \partial_{\eta} f_{t}(x) d \mu(t)+N_{\operatorname{dom} I_{f}}(x)\right\},
$$

but unfortunately such an expression does not hold in general. Formula (4) may fail even for smooth integrands, where $\partial I_{f}(x)$ is nonempty and at the same time $\int_{T} \partial_{\eta} f_{t}(x) d \mu(t)$ is empty (see Example 1).

Our main goal in this paper is to provide general formulae for the (exact) subdifferential and the $\varepsilon$-subdifferential of the convex integral functional $I_{f}$, defined in an arbitrary locally convex space, in the absence of qualification conditions. Other 
characterizations given under appropriate continuity conditions are investigated in [15], while the nonconvex case is treated in [14].

The rest of the paper is organized as follows: In Section 2 we summarize the notation which is needed in the sequel. In Section 3. we give some definitions and preliminary results of vector integration, measurable multifunctions, measurable selections and integral of multifunctions, which are used to study the subdifferential of the integral functional $I_{f}$. In Section 4 , we present our main formulae, which characterize the $\varepsilon$-subdifferential of the integral functional without any qualification conditions (see Theorem 3). In this result, we explore the finite-dimensional reduction approach (see, e.g., [12, 17, 30, 31] and the references therein). Later on, we provide corollaries and simplifications of our main formulae under some qualification conditions of the data (see Corollary 21). General formulae for the discrete sum are derived from Theorem 3 . Finally, in Section 5 we use calculus rules for the $\varepsilon$-subdifferential to get tighter formulae in the setting of Suslin spaces. We also consider there the case of countably discrete measure space to study the subdifferential of series of convex functions (see, e.g., [39]).

\section{Notation}

In this section, we give the main notation and definitions that will be used in the sequel (see, e.g., [3, 4, 18, 25, 29, 36, 42, ).

We denote by $\left(X, \tau_{X}\right)$ a Hausdorff (separated) locally convex space (lcs, for short), endowed with a given (initial) topology $\tau_{X}$, and by $X^{*}$ a locally convex topological dual of $X$ with respect to a given bilinear form $\langle\cdot, \cdot\rangle: X^{*} \times X \rightarrow \mathbb{R}$, defined as

$$
\left\langle x^{*}, x\right\rangle:=\left\langle x, x^{*}\right\rangle:=x^{*}(x) .
$$

Examples of the locally convex topology $\tau_{X^{*}}$ that will be considered on $X^{*}$ are the weak ${ }^{*}$ topology $w\left(X^{*}, X\right)\left(w^{*}\right.$, for short) and the Mackey topology $\tau\left(X^{*}, X\right)$. These two topologies delimit the set of compatible topologies for the pair $\left(X, X^{*}\right)$. Another topology on $X^{*}$ (possibly not compatible for the cited pair) that we will use in the sequel is the strong topology $\beta\left(X^{*}, X\right)$. Bounded sets in $X^{*}$ with respect to the $w^{*}$ or $\beta\left(X^{*}, X\right)$ topologies are the same. The convergence with respect to the weak* topology is denoted by $\rightarrow$. We omit the reference to the topology $\tau_{X}$, and simply write $X$ when no confusion occurs.

For a point $x \in X, \mathcal{N}_{x}\left(\tau_{X}\right)$ represents the family of convex and symmetric neighborhoods of $x$ with respect to the topology $\tau_{X}$. We omit the reference to the used topology and write $\mathcal{N}_{x}$ when there is no confusion. We will write $\overline{\mathbb{R}}:=$ $\mathbb{R} \cup\{-\infty, \infty\}$ and adopt the following conventions,

$$
0 \cdot \infty=0=0 \cdot(-\infty) \text { and } \infty+(-\infty)=(-\infty)+\infty=\infty .
$$

For a seminorm $\rho: X \rightarrow \mathbb{R}, x \in X$, and $r>0$, we denote

$$
B_{\rho}(x, r):=\{y \in X: \rho(x-z) \leq r\} .
$$

For a given function $f: X \rightarrow \overline{\mathbb{R}}$, the (effective) domain of $f$ is

$$
\operatorname{dom} f:=\{x \in X \mid f(x)<+\infty\} .
$$


We say that $f$ is proper if $\operatorname{dom} f \neq \emptyset$ and $f>-\infty$, and inf-compact if for every $\lambda \in \mathbb{R}$ the sublevel set $[f \leq \lambda]:=\{x \in X \mid f(x) \leq \lambda\}$ is compact. We denote by $\Gamma_{0}(X)$ the class of proper lower semicontinuous (lsc) convex functions on $X$. The conjugate of $f$ is the function $f^{*}: X^{*} \rightarrow \overline{\mathbb{R}}$ defined by

$$
f^{*}\left(x^{*}\right):=\sup _{x \in X}\left\{\left\langle x^{*}, x\right\rangle-f(x)\right\}
$$

and the biconjugate of $f$ is the function $f^{* *}: X \rightarrow \overline{\mathbb{R}}$, defined as the restriction of $\left(f^{*}\right)^{*}$ to $X$. For $\varepsilon \geq 0$, the $\varepsilon$-subdifferential of $f$ at a point $x \in X$ where it is finite is the set

$$
\partial_{\varepsilon} f(x):=\left\{x^{*} \in X^{*} \mid\left\langle x^{*}, y-x\right\rangle \leq f(y)-f(x)+\varepsilon, \forall y \in X\right\}
$$

if $f(x)$ is not finite, we set $\partial_{\varepsilon} f(x):=\emptyset$. For a closed linear subspace $F$ of $X$, $\overline{\mathrm{co}}_{F} f: F \rightarrow \overline{\mathbb{R}}$ is the closed convex hull of $f_{\left.\right|_{F}}$, the restriction of $f$ to $F$.

The indicator and the support functions of a set $A \subseteq X$ are, respectively,

$$
\delta_{A}(x):=\left\{\begin{array}{ll}
0 & x \in A \\
+\infty & x \notin A,
\end{array} \quad \sigma_{A}:=\delta_{A}^{*} .\right.
$$

The inf-convolution of $f, g: X \rightarrow \overline{\mathbb{R}}$ is the function

$$
f \square g:=\inf _{z \in X}\{f(z)+g(\cdot-z)\} ;
$$

it is said to be exact at $x$ if there exists $z$ such that $f \square g(x)=f(z)+g(x-z)$.

For a set $A \subseteq X$, we denote by $\operatorname{int}(A), \bar{A}$ (or $\operatorname{cl} A), \operatorname{co}(A)$, and $\overline{\mathrm{co}}(A)$, the interior, the closure, the convex hull, and the closed convex hull of the set $A$, respectively. The linear and the affine hulls of $A$ are respectively defined by

$$
\begin{gathered}
\operatorname{lin}(A):=\operatorname{span}\{A\}:=\cap\{F \subset X: A \subset F, F \text { is linear }\}, \\
\operatorname{aff}(A):=\cap\{F \subset X: A \subset F, F \text { is affine }\} .
\end{gathered}
$$

The relative interior of $A$, denoted by $\operatorname{ri}(A)$, is the interior of $A$ with respect to the trace (or induced) topology on $\operatorname{aff}(A)$, if aff $(A)$ is closed, and the empty set otherwise. Hence, when $X \equiv \mathbb{R}^{n}, \operatorname{ri}(A)$ is the usual relative interior (see [36]). The polar of $A$ is the set

$$
A^{o}:=\left\{x^{*} \in X^{*} \mid\left\langle x^{*}, x\right\rangle \leq 1, \forall x \in A\right\}
$$

and the recession cone of $A$, when $A$ is convex and closed, is the set

$$
A_{\infty}:=\{u \in X \mid x+\lambda u \in A \text { for all } \lambda \geq 0\}
$$

where $x$ is any fixed point in $A$. Finally, the $\varepsilon$-normal set of $A$ at $x$ is $N_{A}^{\varepsilon}(x):=$ $\partial_{\varepsilon} \delta_{A}(x)$. 


\section{Preliminary results}

In what follows, the lcs space $\left(X, \tau_{X}\right)$ and its topological dual $X^{*}$ are defined as in Section 2

A Hausdorff topological space $S$ is said to be a Suslin space if there exist a Polish (i.e., separable completely metrizable) space $P$ and a continuous surjection from $P$ to $S$ (see $7,10,38$ ); hence, $S$ does not need to be metrizable. For example, if $X$ is a separable Banach space, then $(X,\|\cdot\|)$ and $\left(X^{*}, w^{*}\right)$ are Suslin spaces; indeed, the unit ball in $X^{*}$ is weak ${ }^{*}$-separable and weak*-metrizable in that case.

Let $(T, \Sigma, \mu)$ be a nonnegative complete $\sigma$-finite measure space, and let $L^{1}(T, \mathbb{R})$ denote the usual $L^{1}(T)$ space of equivalence classes of (scalar) Lebesgue integrable functions. Given a function $f: T \rightarrow \overline{\mathbb{R}}$, we denote

$$
\mathcal{D}_{f}:=\left\{g \in L^{1}(T, \mathbb{R}): f(t) \leq g(t) \mu \text {-almost everywhere }\right\} .
$$

Definition 1 We define the upper integral of $f$ by

$$
\int_{T}^{*} f(t) d \mu(t):=\inf _{g \in \mathcal{D}_{f}} \int_{T} g(t) d \mu(t)
$$

whenever $\mathcal{D}_{f} \neq \emptyset$, and $\int_{T}^{*} f(t) d \mu(t):=+\infty$, when $\mathcal{D}_{f}=\emptyset$.

Observe that when $f \in L^{1}(T, \mathbb{R})$, the upper integral coincides with the usual Lebesgue integral. Conversely, if $f: T \rightarrow \overline{\mathbb{R}}$ is a measurable (in the usual sense) function with finite upper integrable, then, clearly, $f \in L^{1}(T, \mathbb{R})$ (using the convention that $\left.\inf _{\emptyset}=+\infty\right)$. In other words, for every measurable function $f: T \rightarrow \overline{\mathbb{R}}$ we have that

$$
\int_{T}^{*} f(t) d \mu(t)=\int_{T} f(t) d \mu(t) .
$$

Definition 1 will be used next to introduce the integrability of appropriately measurable (see Definition 2 below) vector-valued functions $f: T \rightarrow X$, via the upper integrability of the scalar-valued functions $\sigma_{B}(f(\cdot))$, for weak* (or, equivalently, Mackey) bounded balanced subsets $B \subseteq X^{*}$. The issue is that these functions are not necessarily measurable, and so we cannot define rigorously their integral. Thus, the upper integral will be involved to overcome this difficulty.

Definition 2 (i) A function $f: T \rightarrow U$, with $U$ being a topological space, is called simple, if there are $k \in \mathbb{N}$, a partition $T_{i} \in \Sigma$ and elements $x_{i} \in U, i=0, \ldots, k$, such that $f(t)=x_{i}$ for $t \in T_{i}$.

(ii) A function $f: T \rightarrow U$, with $U$ being a topological space, is called strongly measurable (measurable, for short), if there exists a sequence $\left(f_{n}\right)_{n}$ of simple functions such that $f(t)=\lim _{n \rightarrow \infty} f_{n}(t)$ for almost every (ae, for short) $t \in T$.

(iii) A strongly measurable function $f: T \rightarrow X$ is said to be strongly integrable (integrable, for short), and we write $f \in \mathcal{L}^{1}(T, X)$, if $\int_{T}^{*} \sigma_{B}(f(t)) d \mu(t)$ is finite for every $\left(\beta\left(X^{*}, X\right)\right)$-bounded balanced subset $B \subseteq X^{*}$.

(iv) A function $f: T \rightarrow X$ is called weakly or scalarly measurable (integrable, resp.), if for every $x^{*} \in X^{*}, t \rightarrow\left\langle x^{*}, f(t)\right\rangle$ is measurable (integrable, resp.). 
A function $f: T \rightarrow X^{*}$ is called $w^{*}$-measurable $\left(w^{*}\right.$-integrable, resp.), if for every $x \in X$, the mapping $t \rightarrow\langle x, f(t)\rangle$ is measurable (integrable, resp.).

(v) We define $\mathcal{L}_{w}^{1}(T, X)$ as the subspace of weakly measurable functions $f$ : $T \rightarrow X$ such that $\int_{T}^{*} \sigma_{B}(f(t)) d \mu(t)$ is finite, for every bounded balanced subset $B \subseteq X^{*}$

We define $\mathcal{L}_{w^{*}}^{1}\left(T, X^{*}\right)$ as the subspace of $\mathrm{w}^{*}$-integrable functions $f$ such that $\int_{T}^{*} \sigma_{B}(f(t)) d \mu(t)$ is finite, for every bounded balanced subset $B \subseteq X$.

(vi) We define $L^{1}(T, X), L_{w}^{1}(T, X)$ and $L_{w^{*}}^{1}\left(T, X^{*}\right)$ as the quotient spaces of $\mathcal{L}^{1}(T, X), \mathcal{L}_{w}^{1}(T, X)$ and $\mathcal{L}_{w^{*}}^{1}\left(T, X^{*}\right)$, respectively, with respect to the equivalence relations $f=g$ ae, $\left\langle f, x^{*}\right\rangle=\left\langle g, x^{*}\right\rangle$ ae $t$ for all $x^{*} \in X^{*}$, and $\langle f, x\rangle=\langle g, x\rangle$ ae $t$ for all $x \in X$, respectively.

(vii) Given $\mathrm{w}^{*}$-integrable function $f: T \rightarrow X^{*}$ and $E \in \Sigma$, the weak integral of $f$ over $E$ is the linear mapping $\int_{E} f(t) d \mu(t):=x_{E}^{\sharp}$, defined on $X$ as

$$
x_{E}^{\sharp}(x):=\int_{E}\langle f(t), x\rangle d \mu(t) .
$$

When $f$ is strongly integrable; hence, $\mathrm{w}^{*}$-integrable, the element $\int_{E} f d \mu$ is also referred to as the strong integral of $f$ over $E$.

(viii) When $X$ is Banach, we define $L^{\infty}(T, X)$ as the normed space (of classes of equivalence with respect to the relation $f=g$ ae) of strongly measurable functions $f: T \rightarrow X$, which are essentially bounded; that is,

$$
\|f\|_{\infty}:=\operatorname{ess} \sup \{\|f(t)\|: t \in T\}<\infty .
$$

(ix) When $X$ is Banach, we call an element $\lambda^{*} \in L^{\infty}(T, X)^{*}$ (the topological dual space of $\left.L^{\infty}(T, X)\right)$ a singular measure, and we write $\lambda^{*} \in L^{\text {sing }}(T, X)$, when there exists a sequence of measurable sets $T_{n}$ such that

$$
T_{n+1} \subseteq T_{n}, \quad \mu\left(T_{n}\right) \rightarrow_{n} 0, \quad \lambda^{*}\left(g \mathbb{1}_{T_{n}^{c}}\right)=0, \text { for all } g \in L^{\infty}(T, X), \text { and all } n \in \mathbb{N},
$$

where $\mathbb{1}_{A}$ denotes the characteristic function of $A$, equal to 1 in $A$ and 0 outside.

In the following remark, we gather some useful comments in order to explain the concepts introduced in the above definition.

Remark 1 (i) If $X$ is a Suslin Banach space and $X^{*}$ is its topological dual, then every $(\Sigma, \mathcal{B}(X))$-measurable function $f: T \rightarrow X$ (that is, $f^{-1}(B) \in \Sigma$ for all $B \in \mathcal{B}(X))$ is weakly measurable. Here, $\mathcal{B}(X)$ is the Borel $\sigma$-Algebra of the open (equivalently, weakly open) sets of $X$ (see, e.g., [10, Theorem III.36 ]).

(ii) In the Banach spaces setting, where $X$ is a Banach space and $X^{*}$ is its topological dual, $L^{1}(T, X)$ coincides with the space of Bochner integrable functions (see, e.g., [16, $\S I I]$ ). If, in addition, $X$ is separable, then both notions of (strong and weak) measurability coincide (see [16, §II, Theorem 2]), and, consequently, $L^{1}(T, X)=L_{w}^{1}(T, X)$ in this case. Similarly, if $\left(X^{*},\|\cdot\|\right)$ is separable, then $L^{1}\left(T, X^{*}\right)=L_{w^{*}}^{1}\left(T, X^{*}\right)$. However, when the Banach space $X$ is separable, but the dual space $X^{*}$ is not (with respect to the dual norm), then strong and weak measurability may not coincide (see [16, §II Example 6]), and so $L^{1}(T, X) \neq L_{w}^{1}(T, X)$ in general. 
(iii) It is clear that every strongly integrable function is weakly integrable. However, the weak measurability of a function $f$ does not necessarily imply the measurability of the function $\sigma_{B}(f(\cdot))$, and so the corresponding integral of this last function is to be understood as the upper integral in the sense of Definition 1.

(iv) In general, the weak integral $\int_{E} f d \mu$ of a w*-integrable function $f: T \rightarrow X^{*}$ on $E \in \Sigma$, may not be in $X^{*}$. But, if $X$ is Banach, then $\int_{E} f d \mu \in X^{*}$, and is referred to in this case as the Gelfand integral of $f$ over $E$ (see [16, §II, Lemma 3.1] and the details therein).

(v) When $X$ is Banach, we know that each functional $\lambda^{*} \in L^{\infty}(T, X)^{*}$ can be uniquely written as

$$
\lambda^{*}(x)=\int_{T}\left\langle\lambda_{1}^{*}(t), x(t)\right\rangle d \mu(t)+\lambda_{2}^{*}(x), \quad \text { for all } x \in L^{\infty}(T, X),
$$

where $\lambda_{1}^{*} \in L_{w^{*}}^{1}\left(T, X^{*}\right), \lambda_{2}^{*} \in L^{\operatorname{sing}}(T, X)$, and the integral defines the duality product between $L_{w^{*}}^{1}\left(T, X^{*}\right)$ and $L^{\infty}(T, X)$ (see, for example, [10, 26]).

We shall need the following decomposability concepts [10, Definition 3, §VII]. Recall that $\mathcal{P}(T)$ is the power set of $T$, and $X^{T}$ is the set of functions from $T$ to $X$.

Definition $3(i)$ Assume that $(T, \Sigma)=(\mathbb{N}, \mathcal{P}(\mathbb{N}))$. A vector space $L \subset X^{T}$ is said to be decomposable if

$$
c_{00}(X):=\left\{\left(x_{n}\right): \exists k_{0} \in \mathbb{N} \text { such that } x_{k}=0, \forall k \geq k_{0}\right\} \subset L .
$$

(ii) Assume that $(T, \Sigma) \neq(\mathbb{N}, \mathcal{P}(\mathbb{N}))$. A vector space $L$ of weakly integrable functions in $X^{T}$ is said to be decomposable if for every $u \in L$, every weakly integrable function $f \in X^{T}$ such that $f(T)$ is relatively compact, and every set $A \in \Sigma$ with finite measure, we have that

$$
f \mathbb{1}_{A}+u \mathbb{1}_{A^{c}} \in L .
$$

Remark 2 The specification of the decomposability above with respect to the underlying $\sigma$-algebra $(T, \Sigma)$ makes sense, since the two definitions may not coincide. For instance, if $X=\mathbb{R}$ and $\mu$ is a finite measure over $(\mathbb{N}, \mathcal{P}(\mathbb{N}))$, then the space $L=c_{00}(X)$ is obviously decomposable in the sense of Definition [3(i), but not with respect to Definition 3(ii). Indeed, the decomposability of $L$ in the sense of Definition 3(ii) would imply that $\ell^{\infty} \subseteq L$.

The above measurability and integrability concepts are extended next to the framework of multifunctions.

Definition 4 A multifunction $G: T \rightrightarrows X$ is called $\Sigma-\mathcal{B}\left(X, \tau_{X}\right)$-measurable (measurable, for simplicity) if its graph, given as

$$
\operatorname{gph} G:=\{(t, x) \in T \times X: x \in G(t)\},
$$

is an element of $\Sigma \otimes \mathcal{B}\left(X, \tau_{X}\right)$. We say that $G$ is weakly measurable if for every $x^{*} \in X^{*}, t \rightarrow \sigma_{G(t)}\left(x^{*}\right)$ is a measurable function. 
The next definition gives the integral of multifunctions in the sense of Aumann (see, for example, [2]).

Definition 5 The strong and the weak integrals of a (non-necessarily measurable) multifunction $G: T \rightrightarrows X^{*}$ are given respectively by

$$
\begin{aligned}
\int_{T} G(t) d \mu(t) & :=\left\{\int_{T} m(t) d \mu(t) \in X^{*}: m \text { is integrable and } m(t) \in G(t) a e\right\}, \\
(w)-\int_{T} G(t) d \mu(t) & :=\left\{\int_{T} m(t) d \mu(t) \in X^{*}: m \text { is } \mathrm{w}^{*} \text {-integrable and } m(t) \in G(t) a e\right\} .
\end{aligned}
$$

In what follows, we fix a function $f: T \times X \rightarrow \overline{\mathbb{R}}$. Given a vector subspace $L$ of $X^{T}$, we shall call an integral functional on $L$, the extended real-valued functional $\hat{I}_{f}$, which is given as

$$
x(\cdot) \in L \rightarrow \hat{I}_{f}(x(\cdot)):=\int_{T}^{*} f(t, x(t)) d \mu(t),
$$

Hence, in particular, when the function $f(\cdot, x(\cdot))$ is measurable, we obtain

$$
\hat{I}_{f}(x(\cdot))=\int_{T} f(t, x(t)) d \mu(t) .
$$

The special but interesting case when $L$ is the linear space of constant functions will deserve a serious study. Since in such a case $\hat{I}_{f}$ can be regarded as a function on $X$, we shall denote it simply as $I_{f}$ and write

$$
x \in X \rightarrow I_{f}(x):=\int_{T}^{*} f(t, x) d \mu(t) .
$$

The assumptions that will be imposed on $f$ will guarantee that $\hat{I}_{f}$ and $I_{f}$ are defined by using the usual integral instead of the upper integral.

The function $f$ is called a $\tau_{X}$-normal integrand (or, simply, normal integral when no confusion occurs), if $f$ is $\Sigma \otimes \mathcal{B}\left(X, \tau_{X}\right)$-measurable and the functions $f(t, \cdot)$ are lsc for ae $t \in T$. In addition, if $f(t, \cdot) \in \Gamma_{0}(X)$ for ae $t \in T$, then $f$ is called convex normal integrand. For simplicity, we denote $f_{t}:=f(t, \cdot)$.

The following result characterizes the Fenchel conjugate of $\hat{I}_{f}$ when $X$ and $X^{*}$ are Suslin spaces, which is known ( [10, Theorem VII-7]), as well as when $(T, \Sigma)=(\mathbb{N}, \mathcal{P}(\mathbb{N}))$.

Proposition 1 Assume that either $X, X^{*}$ are Suslin spaces, or $(T, \Sigma)=(\mathbb{N}, \mathcal{P}(\mathbb{N}))$, and assume that $f$ is a convex normal integrand. Let $L \subset X^{T}$ and $L^{*} \subset X^{* T}$ be two vector spaces of weakly integrable functions, such that $L$ is decomposable and the function

$$
t \rightarrow\langle v(t), u(t)\rangle
$$

is integrable for every $(u, v) \in L \times L^{*}$. Let $\hat{I}_{f}$ be the integral functional defined on $L$. If $f: T \times X \rightarrow \overline{\mathbb{R}}$ is a normal integrand such that $\hat{I}_{f}\left(u_{0}\right)<\infty$ for some $u_{0} \in L$, then the integral functional $\hat{I}_{f^{*}}$ defined on $L^{*}$ satisfies, for all $v \in L^{*}$,

$$
\hat{I}_{f^{*}}(v)=\sup _{u \in L} \int_{T}(\langle u(t), v(t)\rangle-f(t, u(t))) d \mu(t),
$$


Proof First, we may suppose without loss of generality (w.l.o.g.) that $\hat{I}_{f}\left(u_{0}\right) \in \mathbb{R}$; since for otherwise, $\hat{I}_{f}\left(u_{0}\right)=-\infty$ and the conclusion holds trivially. So, the proof in the first case of Suslin spaces follows from [10, Theorem VII-7]. For the proof in the second case when $(T, \Sigma)=(\mathbb{N}, \mathcal{P}(\mathbb{N}))$, we fix $v \in L^{*}$ and denote

$$
\begin{aligned}
\delta(n) & :=\left\langle u_{0}(n), v(n)\right\rangle-f\left(n, u_{0}(n)\right), \\
\alpha & :=\sup _{u \in L} \int_{T}(\langle u(t), v(t)\rangle-f(t, u(t))) d \mu(t) .
\end{aligned}
$$

We consider the sequence $\left(x_{k}\right)_{k}$ defined as

$$
x_{k}(n)=u_{0}(n) \text { for } n>k \text {, and } x_{k}(n):=w_{n} \text { for } n \leq k,
$$

where $w_{n} \in X$ is any vector satisfying

$$
\left\langle w_{n}, v(n)\right\rangle-f\left(n, w_{n}\right) \geq \max \left\{f^{*}(n, v(n))-\frac{1}{k}, \delta(n)\right\}
$$

when $f^{*}(n, v(n))<+\infty$ and, for otherwise,

$$
\left\langle w_{n}, v(n)\right\rangle-f\left(n, w_{n}\right) \geq \max \{k, \delta(n)\} .
$$

Due to the decomposability of the vector space $L$ (see Definition 3)(i)) we see that $\left(x_{k}\right) \in L$. Then, for every $k$ and $k_{0}$ in $\mathbb{N}$ such that $k_{0}<k$ we have

$$
\begin{aligned}
\alpha & \geq \int_{n \leq k_{0}}\left\langle x_{k}(n), v(n)\right\rangle-f\left(n, x_{k}(n)\right) d \mu(n)+\int_{n>k_{0}}\left\langle x_{k}(n), v(n)\right\rangle-f\left(n, x_{k}(n)\right) d \mu(n) \\
& \geq \int_{n \leq k_{0}}\left\langle x_{k}(n), v(n)\right\rangle-f\left(n, x_{k}(n)\right) d \mu(n)+\int_{n>k_{0}} \delta(n) d \mu(n) .
\end{aligned}
$$

Hence, by taking the limit when $k \rightarrow+\infty$ we get

$$
\alpha \geq \int_{n \leq k_{0}} f^{*}(n, v(n)) d \mu(n)+\int_{n>k_{0}} \delta(n) d \mu(n),
$$

and the inequality $\alpha \geq \int_{\mathbb{N}} f^{*}(n, v(n)) d \mu(n)$ follows as $k_{0}$ goes to $+\infty$. This finishes the proof because the converse inequality

$$
\alpha \leq \int_{\mathbb{N}} f^{*}(n, v(n)) d \mu(n)
$$

holds trivially.

We also recall the following result, which gives the representation of the Fenchel conjugate of the integral functional $\hat{I}_{f}$ defined on $L^{\infty}(T, X)$. This result was first proved in [34, Theorem 1] for the case $X=\mathbb{R}^{n}$, and next in [33. Theorem 4] when $X$ is an arbitrary separable reflexive Banach space. We recall that, for $s^{*} \in$ $L^{\text {sing }}(T, X)\left(\subset\left(L^{\infty}(T, X)\right)^{*}\right)$,

$$
\sigma_{\operatorname{dom} \hat{I}_{f}}\left(s^{*}\right):=\sup _{u \in \operatorname{dom} \hat{I}_{f}} s^{*}(u)=\sup _{u \in \operatorname{dom} \hat{I}_{f}}\left\langle s^{*}, u\right\rangle,
$$

where the last product refers to the duality product between $L^{\infty}(T, X)$ and its dual $\left(L^{\infty}(T, X)\right)^{*}$ (we are assuming that $X$ is Banach). 
Theorem 1 Let $X$ be a separable reflexive Banach space, and $f: T \times X \rightarrow \mathbb{R} \cup$ $\{+\infty\}$ be a normal convex integrand. Assume that the integral functional $\hat{I}_{f}$ defined on $L^{\infty}(T, X)$ is finite at some point in $L^{\infty}(T, X)$, and that the integral functional $\hat{I}_{f^{*}}$ defined on $L_{w^{*}}^{1}\left(T, X^{*}\right)$ is finite at some point. Given $u^{*} \in\left(L^{\infty}(T, X)\right)^{*}$, we choose $\ell^{*} \in L_{w^{*}}^{1}\left(T, X^{*}\right)$ and $s^{*} \in L^{\operatorname{sing}}(T, X)$ such that $u^{*}=\ell^{*}+s^{*}$. Then

$$
\left(\hat{I}_{f}\right)^{*}\left(u^{*}\right)=\int_{T} f^{*}\left(t, \ell^{*}(t)\right) d \mu(t)+\sigma_{\operatorname{dom} \hat{I}_{f}}\left(s^{*}\right) .
$$

A straightforward application of the above theorem gives us a representation of the subdifferential of integrand functionals. The proof can be found (for $\varepsilon=0$ ) in [34, Corollary 1B] for the finite-dimensional case, and in [27, Proposition 1.4.1] for arbitrary separable reflexive Banach space. The proof of the general case $\varepsilon \geq 0$ is similar, and is given here for completeness.

Proposition 2 With the assumptions of Theorem 1, for every $u \in L^{\infty}(T, X)$ and $\varepsilon \geq$ 0 , one has that $u^{*}=\ell^{*}+s^{*} \in \partial_{\varepsilon} \hat{I}_{f}(u)$ (with $\ell^{*} \in L_{w^{*}}^{1}\left(T, X^{*}\right)$ and $s^{*} \in L^{\text {sing }}(T, X)$ ) if and only if there exist an integrable function $\varepsilon_{1}: T \rightarrow[0,+\infty)$ and a constant $\varepsilon_{2} \geq 0$ such that

$$
\ell^{*}(t) \in \partial_{\varepsilon_{1}(t)} f(t, u(t)) a e, s^{*} \in N_{\operatorname{dom} \hat{I}_{f}}^{\varepsilon_{2}}(u), \text { and } \int_{T} \varepsilon_{1}(t) d \mu(t)+\varepsilon_{2} \leq \varepsilon .
$$

Proof Take $u^{*}=\ell^{*}+s^{*}$ in $\partial_{\varepsilon} \hat{I}_{f}(u)$; hence, $u \in \operatorname{dom} \hat{I}_{f}$. Then, by Theorem 1 and the definition of $\varepsilon$-subdifferentials, we have

$$
\int_{T}\left(f(t, u(t))+f^{*}\left(t, \ell^{*}(t)\right)-\left\langle\ell^{*}(t), u(t)\right\rangle\right) d \mu(t)+\left(\sigma_{\operatorname{dom} \hat{I}_{f}}\left(s^{*}\right)-\left\langle s^{*}, u\right\rangle\right) \leq \varepsilon .
$$

Hence, we conclude by setting $\varepsilon_{1}(t):=f(t, u(t))+f^{*}\left(t, \ell^{*}(t)\right)-\left\langle\ell^{*}(t), u(t)\right\rangle(\geq 0)$ and $\varepsilon_{2}:=\sigma_{\operatorname{dom} \hat{I}_{f}}\left(s^{*}\right)-\left\langle s^{*}, u\right\rangle(\geq 0)$.

The next result, also given in [34, Theorem 2], will be used in the proof of Theorem 3 below.

Theorem 2 Let $f: T \times \mathbb{R}^{n} \rightarrow \mathbb{R} \cup\{+\infty\}$ be a normal convex integrand. Assume that $\bar{u} \in L^{\infty}\left(T, \mathbb{R}^{n}\right)$, and that for some $r>0$ the function $f(\cdot, \bar{u}(\cdot)+x)$ is integrable for every $x \in \mathbb{R}^{n}$ such that $\|x\|<r$. Then there is some $u^{*}$ in $L^{1}\left(T, \mathbb{R}^{n}\right)$ such that the integral functional $\hat{I}_{f^{*}}$ defined on $L^{1}\left(T, \mathbb{R}^{n}\right)$ satisfies $\hat{I}_{f^{*}}\left(u^{*}\right)<\infty$. Moreover, the integral functional $\hat{I}_{f}$ defined on $L^{\infty}\left(T, \mathbb{R}^{n}\right)$ is continuous (in the $L^{\infty}\left(T, \mathbb{R}^{n}\right)$-norm) at every $u \in L^{\infty}\left(T, \mathbb{R}^{n}\right)$ such that $\|u-\bar{u}\|_{\infty}<r$.

The next result deals with measurable selections in Suslin spaces.

Proposition 3 [10, Theorem III.22] Let $S$ be a Suslin space and $G: T \rightrightarrows S$ be a measurable multifunction with non-empty values. Then there exists a sequence $\left(g_{n}\right)$ of $(\Sigma, \mathcal{B}(S))$-measurable functions such that $\left\{g_{n}(t)\right\}_{n \geq 1}$ is dense in $G(t)$ for every $t \in T$.

Lemma 1 Let $\left(F,\|\cdot\|_{F}\right) \subset X$ be a finite-dimensional Banach subspace, with $\left(F^{*}, \| \cdot\right.$ $\left.\|_{F^{*}}\right)$ being its dual, and denote by $P: X \rightarrow F$ and $P^{*}: F^{*} \rightarrow X^{*}$ the continuous linear projection and its adjoint mapping, respectively. Then there exists a neighborhood $W \subset X$ of 0 (depending only on $P$ and $F$ ) such that, for every integrable function $u^{*}(\cdot): T \rightarrow F^{*}$, the composite function $P^{*} \circ u^{*}(\cdot)$ is integrable and satisfies

$$
\sigma_{W}\left(u^{*}(t) \circ P\right) \leq\left\|u^{*}(t)\right\|_{F^{*}} .
$$


Proof Since the projection mapping $P: X \rightarrow F$ is continuous, there exists a neighborhood $W \in \mathcal{N}_{0}(\tau)$ such that $\|P(x)\|_{F} \leq \sigma_{W^{\circ}}(x)$ for all $x \in X$. Hence,

$$
\sigma_{W}\left(u^{*}(t) \circ P\right)=\sup _{x \in W}\left\langle u^{*}(t), P(x)\right\rangle \leq \sup _{y \in B_{F}(0,1)}\left\langle u^{*}(t), y\right\rangle=\left\|u^{*}(t)\right\|_{F^{*}},
$$

where $B_{F}(0,1)$ is the unit ball in $F$. We are done since the function $P^{*} \circ u^{*}(\cdot)$ inherits the measurability from $u^{*}$.

Lemma 2 Assume that both $X$ and $X^{*}$ are Suslin and let $u^{*}: T \rightarrow X^{*}$ be a weak*measurable function. Set

$$
\mathfrak{G}:=\left\{\left(t, x^{*}, y^{*}, v^{*}\right) \in T \times X^{*} \times X^{*} \times F^{*} \mid x^{*}+y^{*}+P^{*}\left(v^{*}\right)=u^{*}(t)\right\} .
$$

Then $\mathfrak{G} \in \Sigma \otimes \mathcal{B}\left(X^{*} \times X^{*} \times F^{*}\right)$, and for every measurable multifunctions $C_{1}, C_{2}$ : $T \rightrightarrows X^{*}, C_{3}: T \rightrightarrows F^{*}$, the multifunction $C: T \rightrightarrows X^{*} \times X^{*} \times F^{*}$, defined as

$\left(x^{*}, y^{*}, z^{*}\right) \in C(t) \Leftrightarrow\left(x^{*}, y^{*}, z^{*}\right) \in C_{1}(t) \times C_{2}(t) \times C_{3}(t)$ and $u^{*}(t)=x^{*}+y^{*}+P^{*}\left(z^{*}\right)$,

is measurable.

Proof Consider the functions $g$ and $h$ defined as

$$
\begin{gathered}
g\left(t, x^{*}, y^{*}, v^{*}\right)=x^{*}+y^{*}+P^{*}\left(v^{*}\right)-u^{*}(t),\left(t, x^{*}, y^{*}, v^{*}\right) \in T \times X^{*} \times X^{*} \times F^{*}, \\
h\left(x^{*}, y^{*}, v^{*}\right)=x^{*}+y^{*}+P^{*}\left(v^{*}\right),\left(x^{*}, y^{*}, v^{*}\right) \in X^{*} \times X^{*} \times F^{*} .
\end{gathered}
$$

We claim that $g$ is $\left(\Sigma \otimes \mathcal{B}\left(X^{*} \times X^{*} \times F^{*}\right), \mathcal{B}\left(X^{*}\right)\right)$-measurable. First, assume that $u^{*}$ is a simple function; that is, there exists a measurable partition of $T,\left\{T_{i}\right\}_{i=1}^{n}$, and elements $u_{i}^{*} \in X$ such that $u^{*}(t)=\sum u_{i}^{*} \mathbb{1}_{T_{i}}(t)$. Then it is easy to see that, for every open set $U$ on $X^{*}$,

$$
g^{-1}(U)=\bigcup_{i=1}^{n} T_{i} \times h^{-1}\left(U+u_{i}^{*}\right) \in \Sigma \otimes \mathcal{B}\left(X^{*} \times X^{*} \times F^{*}\right) .
$$

Therefore $g$ is $\left(\Sigma \otimes \mathcal{B}\left(X^{*} \times X^{*} \times F^{*}\right), \mathcal{B}\left(X^{*}\right)\right)$-measurable. More generally, since $u^{*}$ is measurable, we can write it as the limit of a sequence of simple functions $u_{n}^{*}$, by [10, Theorem III.36 ]. So, by considering a countable dense set $D$ on $X$ and some $\varepsilon_{n} \rightarrow 0^{+}$, we write

$$
\mathfrak{G}=\bigcap_{v \in D} \bigcap_{n \geq 1} \bigcup_{j \in \mathbb{N}} \bigcap_{k \geq j}\left\{\left(t, x^{*}, y^{*}, v^{*}\right)||\left\langle x^{*}+y^{*}+P^{*}\left(v^{*}\right)-u_{k}^{*}(t), v\right\rangle \mid<\varepsilon_{n}\right\},
$$

and with this we conclude, thanks to the first part of the proof. 


\section{Characterizations via $\varepsilon$-subdifferentials}

In this section, we characterize the $\varepsilon$-subdifferential $(\varepsilon \geq 0)$ of the convex function $I_{f}: X \rightarrow \overline{\mathbb{R}}$, defined in (7) by

$$
I_{f}(x)=\int_{T} f(t, x) d \mu(t)
$$

where $X, X^{*}$ are two lcs paired in duality, and $f: T \times X \rightarrow \overline{\mathbb{R}}$ is a convex normal integrand with respect to $\Sigma \otimes \mathcal{B}\left(X, \tau_{X}\right)$.

We start with the main result of this section, in which we use the following notation, for $x \in X$ and $\eta \geq 0$,

$$
\begin{gathered}
\mathcal{F}(x):=\{L \subseteq X: L \text { is a finite-dimensional linear space and } x \in L\}, \\
\mathcal{I}(\eta):=\left\{\ell \in L^{1}\left(T, \mathbb{R}_{+}\right): \int_{T} \ell(t) d \mu(t) \leq \eta\right\}
\end{gathered}
$$

Theorem 3 For every $x \in X$ and $\varepsilon \geq 0$ we have

$$
\begin{aligned}
\partial_{\varepsilon} I_{f}(x) & =\bigcap_{\substack{L \in \mathcal{F}(x) \\
\begin{array}{r}
\varepsilon=\varepsilon_{1}+\varepsilon_{2} \\
\varepsilon_{1}, \varepsilon_{2} \geq 0 \\
\ell \in \mathcal{I}\left(\varepsilon_{1}\right)
\end{array}}}\left\{\int_{T} \partial_{\ell(t)}\left(f_{t}+\delta_{\mathrm{aff}\left\{L \cap \operatorname{dom} I_{f}\right\}}\right)(x) d \mu(t)+N_{L \cap \operatorname{dom} I_{f}}^{\varepsilon_{2}}(x)\right\} \\
& =\bigcap_{L \in \mathcal{F}(x)} \bigcup_{\ell \in \mathcal{I}(\varepsilon)} \int_{T} \partial_{\ell(t)}\left(f_{t}+\delta_{L \cap \operatorname{dom} I_{f}}\right)(x) d \mu(t)
\end{aligned}
$$

Proof We fix $x \in X$ and $\varepsilon \geq 0$. First, it can be easily checked that the right-hand side in (8) is included in the right-hand side of (9), which is in turn included in $\partial_{\varepsilon} I_{f}(x)$. Thus, if $\partial_{\varepsilon} I_{f}(x)$ is empty, then we are done with the proof of the theorem. In other words, we only need to prove the inclusion " $\subset$ " in (8) when $\partial_{\varepsilon} I_{f}(x)$ is nonempty; hence, $x \in \operatorname{dom} I_{f}$.

We may suppose that $x=0$ (the origin vector in $X$ ). Let us first consider the case when

$$
L \cap \operatorname{dom} I_{f}=\{0\}, \text { for every } L \in \mathcal{F}(0),
$$

which easily leads to $\operatorname{dom} I_{f}=\{0\}$. Then, since $0 \in \operatorname{dom} f_{t}$ for ae $t \in T$, we obtain that

$X^{*}=\partial_{\varepsilon} I_{f}(0)=\int_{T} \partial_{\ell(t)}\left(f_{t}+\delta_{\operatorname{aff}\left\{L \cap \operatorname{dom} I_{f}\right\}}\right)(0) d \mu=\int_{T} \partial_{\ell(t)}\left(f_{t}+\delta_{L \cap \operatorname{dom} I_{f}}\right)(0) d \mu$,

and (8) and (9) hold trivially.

Now, we assume that $L \cap \operatorname{dom} I_{f} \neq\{0\}$, for some $L \in \mathcal{F}(0)$; that is,

$$
\mathcal{F}^{*}(0):=\left\{L \in \mathcal{F}(0): L \cap \operatorname{dom} I_{f} \neq\{0\}\right\} \neq \emptyset .
$$

Observe that for $L \in \mathcal{F}(0)$ being such that $L \cap \operatorname{dom} I_{f}$ is a singleton, as $0 \in \operatorname{dom} I_{f}$ we have that $L \cap \operatorname{dom} I_{f}=\{0\}$, and so

$$
\partial_{\varepsilon}\left(I_{f}+\delta_{\operatorname{span}\left\{L \cap \operatorname{dom} I_{f}\right\}}\right)(0)=\partial_{\varepsilon}\left(I_{f}+\delta_{L \cap \operatorname{dom} I_{f}}\right)(0)=X^{*} .
$$


Consequently, we obtain that

$$
\begin{aligned}
\partial_{\varepsilon} I_{f}(0) & =\bigcap_{L \in \mathcal{F}(0)} \partial_{\varepsilon}\left(I_{f}+\delta_{L \cap \operatorname{dom} I_{f}}\right)(0) \\
& =\bigcap_{L \in \mathcal{F}(0)} \partial_{\varepsilon}\left(I_{f}+\delta_{\operatorname{span}\left\{L \cap \operatorname{dom} I_{f}\right\}}\right)(0) \\
& =\bigcap_{L \in \mathcal{F}^{*}(0)} \partial_{\varepsilon}\left(I_{f}+\delta_{\operatorname{span}\left\{L \cap \operatorname{dom} I_{f}\right\}}\right)(0) .
\end{aligned}
$$

In other words, we only need to characterize the set $\partial_{\varepsilon}\left(I_{f}+\delta_{\operatorname{span}\left\{L \cap \operatorname{dom} I_{f}\right\}}\right)(0)$, for a fixed $L \in \mathcal{F}^{*}(0)$. For this aim we denote

$$
\begin{gathered}
F:=\operatorname{span}\left\{L \cap \operatorname{dom} I_{f}\right\}, \\
\hat{f}(t, z):=f_{\mid T \times F}(t, z),(t, z) \in T \times F,
\end{gathered}
$$

and take $x^{*} \in \partial_{\varepsilon}\left(I_{f}+\delta_{F}\right)(0)$. We also consider a continuous projection $P: X \rightarrow F$, with an adjoint $P^{*}: F^{*} \rightarrow X^{*}$, together with the immersion mapping $i_{F}: F \rightarrow X$ of $F$ in $X$. Then the restriction of $x^{*}$ to $F$, denoted by $x_{\left.\right|_{F}}^{*}$, satisfies for all $z \in F$

$$
\begin{aligned}
\left\langle x_{\left.\right|_{F}}^{*}, z\right\rangle & =\left\langle x^{*}, i_{F}(z)\right\rangle \\
& \leq\left(I_{f}+\delta_{F}\right)\left(i_{F}(z)\right)-\left(I_{f}+\delta_{F}\right)(0)+\varepsilon \\
& =I_{f}\left(i_{F}(z)\right)-I_{f}(0)+\varepsilon \\
& =I_{\hat{f}}(z)-I_{\hat{f}}(0)+\varepsilon ;
\end{aligned}
$$

that is,

$$
x_{\left.\right|_{F}}^{*} \in \partial_{\varepsilon} I_{\hat{f}}(0) .
$$

Because $\operatorname{dom} I_{\hat{f}}=F \cap \operatorname{dom} I_{f} \ni 0$,

$$
\operatorname{span}\left\{\operatorname{dom} I_{\hat{f}}\right\}=\operatorname{aff}\left\{\operatorname{dom} I_{f} \cap L\right\}=\operatorname{span}\left\{\operatorname{dom} I_{f} \cap L\right\}=F,
$$

and $F$ is a finite-dimensional subspace, we know that $I_{\hat{f}}$ is continuous on $\operatorname{ri}\left(\operatorname{dom} I_{\hat{f}}\right)$; that is to say, there exist $\eta>0$ and $x_{0} \in \operatorname{dom} I_{f} \cap L$ such that $x_{0}+\eta \operatorname{co}\left\{ \pm e_{i}\right\} \subseteq$ $\operatorname{dom} I_{\hat{f}}$, where $\left\{ \pm e_{i}\right\}$ is a given basis on $F$. Hence, if $h \in F$ belongs to $\eta \operatorname{co}\left\{ \pm e_{i}\right\}$ we have that $f\left(\cdot, x_{0}+h\right)$ is integrable. So, by applying Theorem 2, we obtain that $\hat{I}_{\hat{f}}$ is continuous in a neighborhood of $x_{0}$ (in $L^{\infty}(T, F)$ ), and the hypotheses of Theorem 1 are satisfied. Then, applying the composition rule to $I_{\hat{f}}$, (10) implies that

$$
x_{\left.\right|_{F}}^{*} \in \partial_{\varepsilon} I_{\hat{f}}(0)=A^{*}\left(\partial_{\varepsilon} \hat{I}_{\hat{f}}(0)\right),
$$

where $A: F \rightarrow L^{\infty}(T, F)$ is given by $A(h)=h \mathbb{1}_{T}$, and $A^{*}: L^{\infty}(T, F)^{*} \rightarrow F^{*}$ is its adjoint defined for $h \in F$ as

$$
A^{*}\left(u^{*}+v^{*}\right)(h)=\int_{T}\left\langle u^{*}(t), h\right\rangle+v^{*}\left(h \mathbb{1}_{T}\right), u^{*} \in L^{1}\left(T, F^{*}\right), v^{*} \in L^{\operatorname{sing}}(T, F) .
$$

Consequently, taking into account Proposition 2, there are $\alpha^{*} \in L^{1}\left(T, F^{*}\right)$ and $\beta^{*} \in L^{\text {sing }}(T, F)$ at the same time as $\varepsilon_{1}, \varepsilon_{2} \geq 0$, with $\varepsilon_{1}+\varepsilon_{2}=\varepsilon$, and $\ell \in \mathcal{I}\left(\varepsilon_{1}\right)$ such that

$$
x_{\left.\right|_{F}}^{*}(h)=\int_{T}\left\langle\alpha^{*}(t), h\right\rangle d \mu(t)+\beta^{*}\left(h \mathbb{1}_{T}\right), \text { for all } h \in F,
$$


and

$$
\alpha^{*}(t) \in \partial_{\ell(t)} \hat{f}_{t}(0) \text { ae, } \beta^{*} \in N_{\operatorname{dom} \hat{I}_{\hat{f}}}^{\varepsilon_{2}}(0) .
$$

Let $z^{*} \in X^{* T}$ and $\lambda^{*} \in \mathbb{R}^{X}$ be defined such that, for ae $t \in T$ and $u \in X$,

$$
z^{*}(t)=P^{*}\left(\alpha^{*}(t)\right)=\alpha^{*}(t) \circ P, \lambda^{*}(u)=\beta^{*}\left(P(u) \mathbb{1}_{T}\right)
$$

Then we verify that $\lambda^{*} \in X^{*}$ and, by Lemma 1, that $z^{*} \in L^{1}\left(T, X^{*}\right)$. Now, from the fact that $\alpha^{*}(t) \in \partial_{\ell(t)} \hat{f}_{t}(0)$, ae, and the definition of $z^{*}$, we obtain that for ae $t \in T$ and all $z \in F$

$$
\begin{aligned}
\left\langle z^{*}(t), z\right\rangle & =\left\langle\alpha^{*}(t), P(z)\right\rangle \\
& \leq \hat{f}_{t}(P(z))-\hat{f}_{t}(0)+\ell(t) \\
& =\left(f_{t}+\delta_{F}\right)(z)-\left(f_{t}+\delta_{F}\right)(0)+\ell(t) .
\end{aligned}
$$

Hence, since the last inequality trivially holds for all $z \in X \backslash F$, we infer that $z^{*}(t) \in \partial_{\ell(t)}\left(f_{t}+\delta_{F}\right)(0)$ ae. Moreover, since $A\left(P\left(\operatorname{dom} I_{f}\right)\right) \subseteq \operatorname{dom} \hat{I}_{\hat{f}}$, by arguing as above we also deduce that $\lambda^{*} \in N_{\operatorname{dom} I_{f} \cap L}^{\varepsilon_{2}}(0)$. Consequently, using (11) it follows that for all $h \in X$

$$
\begin{aligned}
\left(P^{*}\left(x_{\left.\right|_{F}}^{*}\right)\right)(h)=x_{\left.\right|_{F}}^{*}(P(h)) & =\int_{T}\left\langle\alpha^{*}(t), P(h)\right\rangle d \mu(t)+\beta^{*}\left(P(h) \mathbb{1}_{T}\right), \\
& =\int_{T}\left\langle P^{*} \alpha^{*}(t), h\right\rangle d \mu(t)+\beta^{*}\left(P(h) \mathbb{1}_{T}\right), \\
& =\int_{T}\left\langle z^{*}(t), h\right\rangle d \mu(t)+\lambda^{*}(h)
\end{aligned}
$$

showing that

$$
P^{*}\left(x_{\left.\right|_{F}}^{*}\right)=\int_{T} z^{*}(t) d \mu(t)+\lambda^{*} \subset \int_{T} \partial_{\ell(t)}\left(f_{t}+\delta_{F}\right)(0) d \mu(t)+N_{\operatorname{dom} I_{f} \cap L}^{\varepsilon_{2}}(0) .
$$

But we have that $x^{*}-P^{*}\left(x_{\left.\right|_{F}}^{*}\right) \in F^{\perp}$, and so

$$
\begin{aligned}
x^{*} & \in \int_{T} \partial_{\ell(t)}\left(f_{t}+\delta_{F}\right)(0) d \mu(t)+N_{\operatorname{dom} I_{f} \cap L}^{\varepsilon_{2}}(0)+F^{\perp} \\
& =\int_{T} \partial_{\ell(t)}\left(f_{t}+\delta_{F}\right)(0) d \mu(t)+N_{\operatorname{dom} I_{f} \cap L}^{\varepsilon_{2}}(0) .
\end{aligned}
$$

This completes the proof of (8).

Remark 3 As it can be easily seen from the proof of Theorem 3 instead of assuming that $f: T \times X \rightarrow \mathbb{R} \cup\{+\infty\}$ is a normal convex integrand, it is sufficient to suppose that for every finite-dimensional subspace $F$ of $X$, the function $f_{\left.\right|_{F}}: T \times F \rightarrow$ $\mathbb{R} \cup\{+\infty\}$ is a convex normal integrand; of course, both assumptions coincide in the finite-dimensional setting, but they are not equivalent in general. 
Remark 4 It is worth mentioning that Theorem 3 above also holds if, instead of $\mathcal{F}(x)$, we take some subfamily of finite-dimensional subspaces, $\tilde{\mathcal{L}}:=\left\{L_{n}, n \geq 1\right\} \subseteq$ $\mathcal{F}(x)$, such that

$$
\bigcup_{n \in \mathbb{N}} L_{n}=X
$$

For example, if the space $X$ is separable, or more generally, if epi $I_{f}$ is separable, then we take a dense set $\left(x_{i}, \alpha_{i}\right)_{i \geq 1}$ in epi $I_{f}$, and define

$$
L_{n}:=\operatorname{span}\left\{x, x_{i}, i=1, \cdots, n\right\} .
$$

Then it is easy to see that $\bigcap_{n \in \mathbb{N}} \partial_{\varepsilon}\left(I_{f}+\delta_{L_{n}}\right)(x)=\partial_{\varepsilon} I_{f}(x)$.

In Theorem 3 one can weaken the convexity hypothesis by assuming that, for every finite-dimensional subspace $F \subset X, f_{\left.\right|_{F}}$ is a normal integrand and

$$
\overline{\mathrm{Co}}_{F} I_{f}=I_{\overline{\mathrm{co}}_{F} f} \text {. }
$$

Corollary 1 Under condition (12), we have that

$$
\begin{gathered}
\partial_{\varepsilon} I_{f}(x) \subseteq \bigcap_{\begin{array}{r}
L \in \mathcal{F}(x) \\
\begin{array}{r}
\varepsilon=\varepsilon_{1}+\varepsilon_{2} \\
\varepsilon_{1}, \varepsilon_{2} \geq 0 \\
\ell \in \mathcal{I}\left(\varepsilon_{1}\right)
\end{array}
\end{array}}\left\{\int_{T} \partial_{\ell(t)+m_{x, L}(t)}\left(f_{t}+\delta_{\mathrm{aff}\left\{L \cap \operatorname{dom} I_{f}\right\}}\right)(x) d \mu(t)\right. \\
\left.+N_{\operatorname{dom} I_{f} \cap L}^{\varepsilon_{2}}(x)\right\}, \\
\partial_{\varepsilon} I_{f}(x) \subseteq \bigcap_{L \in \mathcal{F}(x)} \bigcup_{\ell \in \mathcal{I}(\varepsilon)}\left\{\int_{T} \partial_{\ell(t)+m_{x, L}(t)}\left(f_{t}+\delta_{L \cap \operatorname{dom} I_{f}}\right)(x) d \mu(t)\right\},
\end{gathered}
$$

where

$$
m_{x, L}(\cdot):=f(\cdot, x)-\overline{\mathrm{co}}_{L} f(\cdot, x)
$$

is the modulus of convexity over $F$. In addition, if $\partial_{\varepsilon} I_{f}(x) \neq \emptyset$, then

$$
\int_{T} m_{x, L}(t) d \mu(t) \leq \varepsilon, \text { for all } L \in \mathcal{F}(x) .
$$

Proof The first statement being straightforward from Theorem 3, we only prove the last one. Take $L \in \mathcal{F}(x)$. Then, for every $t \in T$, we have that

$$
f(t, x) \geq \overline{\mathrm{CO}}\left(f(t, \cdot)_{\left.\right|_{L}}\right)(x) \geq \overline{\mathrm{CO}} f(t, x) .
$$

Also, the non-emptiness of $\partial_{\varepsilon} I_{f}(x)$ ensures that $I_{f}(x) \leq \overline{\mathrm{co}} I_{f}(x)+\varepsilon$, so that the hypothesis $I_{\overline{\mathrm{co}} f}(x)=\overline{\mathrm{Co}} I_{f}(x)$ leads to $I_{f}(x) \leq I_{\overline{\mathrm{co}} f}(x)+\varepsilon$. This implies that

$$
\int_{T}\left(f(t, x)-\overline{\mathrm{co}}\left(f(t, \cdot)_{\left.\right|_{L}}\right)(x)\right) d \mu(t) \leq \varepsilon
$$

In particular, if $f$ is a convex normal integrand, or if $\partial I_{f}(x) \neq \emptyset$, then we get $m_{x, L}(\cdot)=0$. 
The next example, given for smooth functions $f_{t}$ defined on finite-dimensional spaces, justifies the use of the indicator function inside the integral symbol in the formulae of Theorem 3 .

Example 1 Consider the function $f(x):=\frac{b}{a} x+b+\delta_{[-\eta, \eta]}(x), a, b, \eta>0$. Then we have

$$
\partial_{\varepsilon} f(0)=\left[-\frac{\varepsilon}{\eta}+\frac{b}{a}, \frac{\varepsilon}{\eta}+\frac{b}{a}\right] .
$$

We consider the Lebesgue measure on $] 0,1]$ and the convex normal integrand $f:] 0,1] \times \mathbb{R} \rightarrow[0,+\infty]$ given by

$$
f(t, x):=\frac{b(t)}{a(t)} x+b(t)+\delta_{[-\eta(t), \eta(t)]}(x),
$$

where $a(t)=\eta(t)=t$ and $b(t)=\frac{1}{\sqrt{t}}+1$. Hence,

$$
I_{f}(x)=\left\{\begin{array}{ccc}
\int_{0}^{1}\left(1+\frac{1}{\sqrt{t}}\right) d t & \text { if } & x=0 \\
+\infty & \text { if } & x \neq 0
\end{array}\right.
$$

and we obtain $\partial I_{f}(0)=\mathbb{R}$, while

$$
\partial_{\varepsilon} f_{t}(0)=\left[-\frac{\varepsilon}{t}+\frac{\frac{1}{\sqrt{t}}+1}{t}, \frac{\varepsilon}{t}+\frac{\frac{1}{\sqrt{t}}+1}{t}\right]=\left[\frac{1-\varepsilon}{t}+\frac{1}{t^{3 / 2}}, \frac{1+\varepsilon}{t}+\frac{1}{t^{3 / 2}}\right] .
$$

Consequently, the set $\int_{0}^{1} \partial_{\varepsilon} f_{t}(0) d t$ is empty for every $0<\varepsilon<1$.

Remark 5 Observe that the formulae of Theorem 3 can be simplified if some qualification conditions (QC, for short) are in force. For instance, each one of conditions QC(i)- $\mathrm{QC}(\mathrm{iv})$ below (see [42, Theorem 2.8.3]) ensures the validity of the exact sum rule

$$
\partial_{\varepsilon}\left(f_{t}+\delta_{\mathrm{aff}\left(L \cap \operatorname{dom} I_{f}\right)}\right)(x)=\partial_{\varepsilon} f_{t}(x)+\partial \delta_{\mathrm{aff}\left(L \cap \operatorname{dom} I_{f}\right)}(x), t \in T, L \in \mathcal{F}(x),
$$

which in turn gives rise to characterizations of $\partial_{\varepsilon} I_{f}(x)$ by means only of the $\varepsilon$ subdifferentials of the $f_{t}$ 's:

QC(i) $X=\mathbb{R}^{n}$ and $\operatorname{ri}\left(\operatorname{dom} f_{t}\right) \cap \operatorname{aff}\left(L \cap \operatorname{dom} I_{f}\right) \neq \emptyset$.

QC(ii) $X$ Banach and $\mathbb{R}_{+}\left(\operatorname{dom} f_{t}-\operatorname{aff}\left(\operatorname{dom} I_{f} \cap L\right)\right)$ is a closed subspace.

QC(iii) $f_{t}$ is continuous at some point of $\operatorname{dom} I_{f}$.

QC(iv) For every $B \in \mathcal{N}_{0}$, there exist $r>0$ and $V \in \mathcal{N}_{0}$ such that

$$
V \cap \operatorname{span}\left\{\operatorname{dom} f_{t}-\operatorname{aff}\left(\operatorname{dom} I_{f} \cap L\right)\right\} \subseteq\left\{f_{t} \leq r\right\} \cap B-\operatorname{aff}\left(\operatorname{dom} I_{f} \cap L\right) .
$$

All of the above conditions imply the following property (see, e.g., [6, 8, 11, 13]):

$\mathrm{QC}(\mathrm{v})$ For every $x^{*} \in X^{*}$,

$$
\left(f_{t}+\delta_{\mathrm{aff}\left(\operatorname{dom} I_{f} \cap L\right)}\right)^{*}\left(x^{*}\right)=\min \left\{f_{t}^{*}\left(y^{*}\right): x^{*}-y^{*} \in\left(\operatorname{aff}\left(\operatorname{dom} I_{f} \cap L\right)\right)^{\circ}\right\}
$$


Corollary 2 In the setting of Theorem [3, suppose that one of conditions $Q C(i)$ to $Q C(v)$ holds in a measurable set $T_{0} \subset T$. Then for all $x \in X$

$$
\begin{aligned}
\partial I_{f}(x)=\bigcap_{L \in \mathcal{F}(x)}\left\{\int_{T_{0}}\left(\partial f_{t}(x)+\left(\operatorname{aff}\left(\operatorname{dom} I_{f} \cap L-x\right)\right)^{\perp}\right) d \mu(t)\right. \\
\left.\quad+\int_{T_{0}^{c}} \partial\left(f_{t}+\delta_{\operatorname{aff}\left\{L \cap \operatorname{dom} I_{f}\right\}}\right)(x) d \mu(t)+N_{L \cap \operatorname{dom} I_{f}}(x)\right\}
\end{aligned}
$$

where $T_{0}^{c}$ is the complement of $T_{0}$. In particular, if $T$ is finite, then

$$
\partial\left(\sum_{t \in T} f_{t}\right)(x)=\bigcap_{\varepsilon>0} \mathrm{cl}^{w^{*}}\left\{\sum_{t \in T_{0}} \partial f_{t}(x)+\sum_{t \in T_{0}^{c}} \partial_{\varepsilon} f_{t}(x)\right\} .
$$

Proof Equation (14) is direct from Theorem 3 and Remark 5, and so, we only need to prove (15). Fix $x \in X, V \in \mathcal{N}_{0}$ and choose $L \in \mathcal{F}(x)$ such that $L^{\perp} \subseteq V$. We may assume that $\partial I_{f}(x) \neq \emptyset$. By (14), and taking into account [19, Theorem 3.1], we have, for every $\varepsilon>0$,

$$
\begin{aligned}
\partial\left(\sum_{i \in T} f_{i}\right)(x) \subseteq & \sum_{i \in T_{0}} \partial f_{i}(x)+\left(\operatorname{aff}\left(\operatorname{dom} I_{f} \cap L-x\right)\right)^{\perp} \\
& +\sum_{i \in T_{0}^{c}} \partial\left(f_{t}+\delta_{\operatorname{aff}\left\{L \cap \operatorname{dom} I_{f}\right\}}\right)(x)+N_{L \cap \operatorname{dom} I_{f}}(x) \\
\subseteq & \sum_{i \in T_{0}} \partial f_{i}(x)+\left(\operatorname{aff}\left(\operatorname{dom} I_{f} \cap L-x\right)\right)^{\perp}+\sum_{i \in T_{0}^{c}} \partial_{\varepsilon} f_{t}(x) \\
& +\left(\operatorname{aff}\left(\operatorname{dom} I_{f} \cap L-x\right)\right)^{\perp}+N_{L \cap \operatorname{dom} I_{f}}(x)+V
\end{aligned}
$$

hence, $\partial f_{i}(x) \neq \emptyset$ for all $i \in T_{0}$. But we have

$\left(\operatorname{aff}\left(\operatorname{dom} I_{f} \cap L-x\right)\right)^{\perp}+\left(\operatorname{aff}\left(\operatorname{dom} I_{f} \cap L-x\right)\right)^{\perp}+N_{L \cap \operatorname{dom} I_{f}}(x) \subseteq N_{L \cap \operatorname{dom} I_{f}}(x)$,

and so

$$
\partial\left(\sum_{i \in T} f_{i}\right)(x) \subseteq \sum_{i \in T_{0}} \partial f_{i}(x)+\sum_{i \in T_{0}^{c}} \partial_{\varepsilon} f_{t}(x)+N_{L \cap \operatorname{dom} I_{f}}(x)+V .
$$

Moreover, since (see [17, Lemma 11])

$$
N_{L \cap \operatorname{dom} I_{f}}(x)=\left[\operatorname{cl}\left(\sum_{i \in T_{0}} \partial f_{i}(x)+\sum_{i \in T_{0}^{c}} \partial_{\varepsilon} f_{t}(x)+L^{\perp}\right)\right]_{\infty},
$$

it follows that

$$
\partial\left(\sum_{i \in T} f_{i}\right)(x) \subseteq \sum_{i \in T_{0}} \partial f_{i}(x)+\sum_{i \in T_{0}^{c}} \partial_{\varepsilon} f_{t}(x)+V+V
$$


which in turn implies that

$$
\begin{aligned}
\partial\left(\sum_{i \in T} f_{i}\right)(x) & \subseteq \bigcap_{\varepsilon>0, i \in T_{0}^{c}} \bigcap_{V \in \mathcal{N}_{0}}\left[\sum_{i \in T_{0}} \partial f_{i}(x)+\sum_{i \in T_{0}^{c}} \partial_{\varepsilon} f_{t}(x)+V+V\right] \\
& =\bigcap_{\varepsilon>0, i \in T_{0}^{c}} \operatorname{cl}\left\{\sum_{i \in T_{0}} \partial f_{i}(x)+\sum_{i \in T_{0}^{c}} \partial_{\varepsilon} f_{t}(x)\right\} .
\end{aligned}
$$

This yields the direct inclusion " $\subset$ " and then completes the proof, since the opposite inclusion is easily checked.

Corollary 3 Let $f: T \times \mathbb{R}^{n} \rightarrow \overline{\mathbb{R}}$ be a convex normal integrand. Assume that $\mathrm{ri}\left(\operatorname{dom} f_{t}\right) \cap$ $\operatorname{aff}\left(\operatorname{dom} I_{f}\right) \neq \emptyset$ for almost all $t \in T$. Then for every $x \in \mathbb{R}^{n}$

$$
\partial I_{f}(x)=\int_{T}\left(\partial f_{t}(x)+N_{\operatorname{dom} I_{f}}(x)\right) d \mu(t) .
$$

Proof Fix $x \in \mathbb{R}^{n}$. Using Corollary 2, we have that

$$
\begin{aligned}
\partial I_{f}(x) & =\int_{T}\left(\partial f_{t}(x)+\left(\operatorname{aff}\left(\operatorname{dom} I_{f}-x\right)\right)^{\perp}\right) d \mu(t)+N_{\operatorname{dom} I_{f}}(x) \\
& =\int_{T}\left(\partial f_{t}(x)+\left(\operatorname{aff}\left(\operatorname{dom} I_{f}-x\right)\right)^{\perp}+N_{\operatorname{dom} I_{f}}(x)\right) d \mu(t) \\
& =\int_{T}\left(\partial f_{t}(x)+N_{\operatorname{dom} I_{f}}(x)\right) d \mu(t) .
\end{aligned}
$$

Remark 6 Example 1 shows that (16) cannot be simplified to

$$
\partial I_{f}(x)=\int_{T} \partial f_{t}(x) d \mu(t)+N_{\operatorname{dom} I_{f}}(x) .
$$

Example 2 The main feature of the finite sum given in (15) is that the characterization of $\partial I_{f}(x)$ does not involve the normal cone $N_{\mathrm{dom} I_{f} \cap L}(x)$. This fact is specific to this finite case and cannot be true in general, even for smooth data functions $f_{t}$ with $\int_{T} \partial f_{t}(x) d \mu(t) \neq \emptyset$. For example, consider the Lebesgue measure on $] 0,1]$ and the integrand $f:] 0,1] \times \mathbb{R} \rightarrow \mathbb{R}$ given by

$$
f(t, x)=x^{2} / t
$$

Then we obtain $I_{f}=\delta_{\{0\}}$ and, so, $\partial f_{t}(0)=\{0\}$, while $\partial I_{f}(0)=\mathbb{R}$. The same example can be adapted to construct a counterexample for a countable measure over the measurable space $(\mathbb{N}, \mathcal{P}(\mathbb{N}))$.

Next, we give another formula for the subdifferential of finite sums of convex functions, where a qualification condition involving the relative interiors is satisfied by only a part of the family $\left\{f_{t}, t \in T\right\}$. We need the following technical Lemma, which is an adaptation of classical techniques in the finite-dimension setting (see, e.g., [42, Corollary 2.3.5]). 
Lemma 3 Let $g \in \Gamma_{0}(X)$ and let $L \subset X$ be a finite-dimensional affine subspace. If $g$ is continuous relative to $\operatorname{aff}(\operatorname{dom} g)$ at some point in $\operatorname{dom} g \cap L$, then for every $x^{*} \in X^{*}$

$$
\left(g+\delta_{L}\right)^{*}\left(x^{*}\right)=\min \left\{g^{*}\left(y^{*}\right)+\delta_{L}^{*}\left(x^{*}-y^{*}\right): y^{*} \in X^{*}\right\} \text {. }
$$

Corollary 4 Assume that $T$ is finite and let $\left\{f_{t}\right\}_{t \in T} \subseteq \Gamma_{0}(X)$ and $T_{0} \subset T$ be given. Then the following statements hold true:

(i) If $\bigcap_{t \in T_{0}} \operatorname{ri}_{\text {aff }\left(\operatorname{dom} f_{t}\right)}\left(\operatorname{dom} f_{t}\right) \cap \bigcap_{t \in T_{0}^{c}} \operatorname{dom} f_{t} \neq \emptyset$ and each $f_{t}$ with $t \in T_{0}$ is continuous on $\operatorname{ri}_{\text {aff }\left(\operatorname{dom} f_{t}\right)}\left(\operatorname{dom} f_{t}\right)$, then for every $x \in X$

$$
\partial\left(\sum_{t \in T} f_{t}\right)(x)=\bigcap_{\varepsilon>0} \mathrm{cl}^{w^{*}}\left\{\sum_{t \in T_{0}} \partial f_{t}(x)+\sum_{t \in T_{0}^{c}} \partial_{\varepsilon} f_{t}(x)\right\}
$$

(ii) If $\bigcap_{t \in T_{0}} \operatorname{int}\left(\operatorname{dom} f_{t}\right) \cap \bigcap_{t \in T_{0}^{c}} \operatorname{dom} f_{t} \neq \emptyset$ and each $f_{t}$ with $t \in T_{0}$ is continuous on $\operatorname{int}\left(\operatorname{dom} f_{t}\right)$, then for every $x \in X$

$$
\partial\left(\sum_{t \in T} f_{t}\right)(x)=\sum_{t \in T_{0}} \partial f_{t}(x)+\bigcap_{\varepsilon>0} \mathrm{cl}^{w^{*}}\left\{\sum_{t \in T_{0}^{c}} \partial_{\varepsilon} f_{t}(x)\right\} .
$$

Proof Let $f:=\sum_{t \in T} f_{t}$. Fix $t \in T_{0}$ and consider $L \in \mathcal{F}(x)$. Then, by applying Lemma 3 with $g=f_{t}$ and $L=\operatorname{aff}(\operatorname{dom} f \cap L)$, we ensure the validity of condition (13). Therefore statement (i) follows by applying Corollary 2. Statement (ii) follows from (i) by arguing as in Lemma 3.

\section{Suslin spaces or discrete measure space}

In this section, we give more sharp characterizations of the $\varepsilon$-subdifferential of $I_{f}$ under the cases where either $X, X^{*}$ are Suslin spaces, or $(T, \Sigma)=(\mathbb{N}, \mathcal{P}(\mathbb{N}))$. These settings, indeed, permit the use of measurable selection theorems, which give us more control over the integration of the multifunctions $\partial_{\varepsilon(t)} f_{t}(x)$ and $N_{\operatorname{dom} I_{f} \cap L}(x)$. We recall that $f: T \times X \rightarrow \mathbb{R} \cup\{+\infty\}$ is a given normal convex integrand, and $(T, \Sigma, \mu)$ is a nonnegative complete $\sigma$-finite measure space. The function $I_{f}: X \rightarrow$ $\mathbb{R} \cup\{+\infty\}$ is defined as

$$
I_{f}(x)=\int_{T} f(t, x) d \mu(t) .
$$

The following corollary rewrites the characterization given in Theorem 3 by using only the $\varepsilon$-subdifferential of the $f_{t}$ 's.

Theorem 4 We suppose that either $X$ and $X^{*}$ are Suslin spaces or $(T, \Sigma)=(\mathbb{N}, \mathcal{P}(\mathbb{N}))$. Then for every $x \in X$ and $\varepsilon \geq 0$ we have

$$
\partial_{\varepsilon} I_{f}(x)=\bigcap_{\substack{L \in \mathcal{F}(x) \varepsilon_{1}, \varepsilon_{2} \geq 0 \\ \varepsilon=\varepsilon_{1}+\varepsilon_{2} \\ \ell \in \mathcal{I}\left(\varepsilon_{1}\right)}} \bigcap_{\eta \in L^{1}\left(T, \mathbb{R}_{+}^{*}\right)} \mathrm{cl}^{\beta\left(X^{*}, X\right)}\left\{\int_{T}\left(\partial_{\ell(t)+\eta(t)} f_{t}(x)+N_{\operatorname{dom} I_{f} \cap L}^{\varepsilon_{2}}(x)\right) d \mu(t)\right\} .
$$


Proof We only need to prove the inclusion " $\subseteq$ " in which we suppose that $x=0$. Take $F \in \mathcal{F}(0), \varepsilon>0$ and

$$
L:=\operatorname{span}\left\{F \cap \operatorname{dom} I_{f}\right\}\left(=\operatorname{aff}\left\{F \cap \operatorname{dom} I_{f}\right\}\right),
$$

say $L=\operatorname{span}\left\{e_{i}\right\}_{1}^{p}$, with $\left\{e_{i}\right\}_{1}^{p}$ being linearly independent, and $\operatorname{co}\left\{ \pm e_{i}\right\}_{i=1}^{p}$ is the unit closed ball in $L$ with respect to a norm $\|\cdot\|_{L}$ (on $L$ ). Let $P: X \rightarrow L$ be a continuous projection and let $W \in \mathcal{N}_{0}$ be as in Lemma 1, Given $\delta>0$, we pick an integrable function $\gamma: T \rightarrow(0,+\infty)$ such that

$$
\int_{T} \gamma(t) d \mu \leq \delta
$$

and define the measurable multifunctions $U, V: T \rightrightarrows L^{*}$ as

$$
\begin{aligned}
U(t) & :=\left\{x^{*} \in X^{*}:\left|\left\langle x^{*}, e_{i}\right\rangle\right| \leq \gamma(t), i=1, \ldots, p\right\}, \\
V(t) & :=\left\{x^{*} \in L^{*}:\left|\left\langle x^{*}, e_{i}\right\rangle\right| \leq \gamma(t), i=1, \ldots, p\right\} .
\end{aligned}
$$

Now, take $x^{*} \in \partial_{\varepsilon} I_{f}(0)$ and fix a positive measurable function $\eta$. By formula (8) in Theorem 3 there exist $\varepsilon_{1}, \varepsilon_{2} \geq 0$ with $\varepsilon_{1}+\varepsilon_{2}=\varepsilon, \ell \in \mathcal{I}\left(\varepsilon_{1}\right)$, an integrable selection $x_{L, \varepsilon}^{*}(\cdot)$ of the multifunction $t \rightarrow \partial_{\ell(t)}\left(f_{t}+\delta_{L}\right)(0)$, and $\lambda^{*} \in N_{\operatorname{dom} I_{f} \cap L}^{\varepsilon_{2}}(0)$ such that

$$
x^{*}=\int_{T} x_{L, \varepsilon}^{*}(t) d \mu(t)+\lambda^{*} .
$$

Since $U(t) \in \mathcal{N}_{0}$, by [19, Theorems 3.1 and 3.2] we have that, for ae $t \in T$,

$$
\begin{aligned}
x_{L, \varepsilon}^{*}(t) \in \partial_{\ell(t)}\left(f_{t}+\delta_{L}\right)(0) & \subset \partial_{\ell(t)+\eta(t)} f_{t}(0)+L^{\perp}+U(t) \\
& \subset \partial_{\ell(t)+\eta(t)} f_{t}(0)+N_{\text {dom } I_{f} \cap L}(0)+P^{*}(V(t)) .
\end{aligned}
$$

We define the multifunction $G: T \rightrightarrows X^{*} \times X^{*} \times L^{*}$ as

$$
\left(y^{*}, w^{*}, v^{*}\right) \in G(t) \Leftrightarrow\left\{\begin{array}{c}
y^{*} \in \partial_{\ell(t)+\eta(t)} f(t, 0), w^{*} \in N_{\mathrm{dom} I_{f} \cap L}(0), \text { and } v^{*} \in V(t), \\
x_{L, \varepsilon}^{*}(t)=y^{*}+w^{*}+P^{*}\left(v^{*}\right) .
\end{array}\right.
$$

If $X, X^{*}$ are Suslin spaces, then, by Lemma $2 G$ is measurable, and so, by Proposition 3 it admits a measurable selection $\left(y^{*}(\cdot), w^{*}(\cdot), v^{*}(\cdot)\right)$. This also obviously holds when $(T, \Sigma)=(\mathbb{N}, \mathcal{P}(\mathbb{N}))$. Thus, by Lemma 1 the function $u^{*}(t):=v^{*}(t) \circ P$ is integrable and we get

$$
\sigma_{W}\left(u^{*}(t)\right) \leq \max _{i=1, \ldots, p}\left\langle v^{*}(t), e_{i}\right\rangle \leq \gamma(t) \text { for ae } t \in T
$$

Consequently, the function $y^{*}+w^{*}=x_{L, \varepsilon}^{*}(\cdot)-u^{*}(\cdot)$ is strongly integrable and we have (recall (17))

$$
\begin{aligned}
\sigma_{W}\left(x^{*}-\int_{T}\left(y^{*}(t)+w^{*}(t)\right) d \mu(t)-\lambda^{*}\right) & =\sigma_{W}\left(\int_{T} x_{L, \varepsilon}^{*}(t) d \mu(t)-\int_{T}\left(y^{*}(t)+w^{*}(t)\right) d \mu(t)\right) \\
& =\sigma_{W}\left(\int_{T} u^{*}(t) d \mu(t)\right) \\
& \leq \int_{T} \sigma_{W}\left(u^{*}(t)\right) d \mu(t) \\
& \leq \int_{T} \gamma(t) d \mu \leq \delta
\end{aligned}
$$


that is,

$$
x^{*}-\int_{T}\left(y^{*}(t)+w^{*}(t)\right) d \mu(t)-\lambda^{*} \in \delta W^{\circ},
$$

and, due to the arbitrariness of $\delta$,

$$
x^{*} \in \mathrm{cl}^{\beta\left(X^{*}, X\right)}\left(\int_{T}\left(\partial_{\ell(t)+\eta(t)} f_{t}(0)+N_{\mathrm{dom}} I_{f} \cap L(0)\right) d \mu(t)+N_{\operatorname{dom} I_{f} \cap L}^{\varepsilon_{2}}(0)\right) .
$$

Finally, since $N_{\operatorname{dom} I_{f} \cap L}^{\varepsilon_{2}}(0) \subset \int_{T} N_{\operatorname{dom} I_{f} \cap L}^{\varepsilon_{2}}(0) d \mu(t)$ we conclude that

$$
x^{*} \in \mathrm{cl}^{\beta\left(X^{*}, X\right)} \int_{T}\left(\partial_{\ell(t)+\eta(t)} f_{t}(0)+N_{\operatorname{dom} I_{f} \cap L}^{\varepsilon_{2}}(0)\right) d \mu(t) .
$$

The next result is a finite-dimensional-like characterization of the subdifferential of $I_{f}$. Recall that a closed affine subspace $A \subset X$ is said to have a continuous projection if there exists an affine continuous projection from $X$ to $A$, or equivalently, if there exists a continuous linear projection from $X$ to $A-x_{0}$, where $x_{0} \in A$.

Theorem 5 Let $X, X^{*}$ and $T$ be as in Theorem 4. If $I_{f}$ is continuous on $\operatorname{ri}\left(\operatorname{dom} I_{f}\right) \neq$ $\emptyset$ and $\overline{\operatorname{aff}}\left(\operatorname{dom} I_{f}\right)$ has a continuous projection, then

$$
\partial I_{f}(x)=\bigcap_{\eta \in L^{1}\left(T, \mathbb{R}_{+}^{*}\right)} \mathrm{cl}^{w^{*}}\left\{(w)-\int_{T}\left(\partial_{\eta(t)} f_{t}(x)+N_{\operatorname{dom} I_{f}}(x)\right) d \mu(t)\right\} .
$$

Proof Because the inclusion " $\subseteq$ " is immediate we only need to prove the other inclusion " $\subseteq$ " when $x=0$; hence, $F:=\overline{\operatorname{aff}}\left(\operatorname{dom} I_{f}\right)$ is a closed subspace of $X$. Let $x^{*} \in \partial I_{f}(0), \eta \in L^{1}(T,(0,+\infty))$, and $V:=\left\{h^{*} \in X^{*}:\left|\left\langle h^{*}, e_{i}\right\rangle\right| \leq 1, \quad i=1, \ldots, p\right\}$ for some $\left\{e_{i}\right\}_{i=1}^{p} \subset X$. By the current assumption, we take $x_{0} \in \operatorname{ri}\left(\operatorname{dom} I_{f}\right)$ and a continuous projection $P: X \rightarrow F$. Define $L=\operatorname{span}\left\{e_{i}, P\left(e_{i}\right), x_{0}\right\}_{i=1}^{p}$ and

$$
W(t):=\left\{h^{*} \in X^{*}: \max \left\{\left|\left\langle h^{*}, e_{i}\right\rangle\right|,\left|\left\langle h^{*}, P\left(e_{i}\right)\right\rangle\right|,\left|\left\langle h^{*}, x_{0}\right\rangle\right|\right\} \leq \varepsilon(t), i=1, \ldots, p\right\},
$$

where $\varepsilon(\cdot)$ is any positive integrable function with values on $(0,1)$ and $\int_{T} \varepsilon d \mu \leq 1 / 2$. Then

$$
L^{\perp}+W(t) \subseteq W(t) \subseteq V .
$$

Because $L \cap \operatorname{ri}\left(\operatorname{dom} I_{f}\right) \neq \emptyset$, we have (see, e.g., Corollary 4$)$

$$
N_{\mathrm{dom} I_{f} \cap L}(0)=\mathrm{cl}^{w^{*}}\left(L^{\perp}+N_{\operatorname{dom} I_{f}}(0)\right) .
$$

By Theorem 4 there exists a (strong) integrable selection $y^{*}(t) \in \partial_{\eta(t)} f_{t}(0)+$ $N_{\operatorname{dom} I_{f} \cap L}(0) \subset \partial_{\eta(t)} f_{t}(0)+N_{\operatorname{dom} I_{f}}(0)+W(t)$, due to (19), such that

$$
x^{*}-\int_{T} y^{*} d \mu \in V .
$$

Also, by the measurability of multifunctions $\partial_{\eta(\cdot)} f \cdot(0), N_{\operatorname{dom} I_{f}}(0)$, and $W(\cdot)$ (see, e.g., [23] ), there exists a (weakly) measurable selection $z^{*}(\cdot)$ of $\partial_{\eta(\cdot)} f \cdot(0)+N_{\operatorname{dom} I_{f}}(0)$ such that

$$
y^{*}(t)-z^{*}(t) \in W(t) \text { for ae }
$$


(the existence of such a selection is guaranteed for Suslin spaces by the representation theorem of Castaing, while it is straightforward in the discrete case).

Let us verify that the function $z^{*}(\cdot) \circ P$ is weakly integrable: Given $U \in \mathcal{N}_{0}$ such that $x_{0}+P(U) \subset \operatorname{ri}\left(\operatorname{dom} I_{f}\right)$ (using the the continuity of $P$ ) we have, for every $y \in U$,

$$
\begin{aligned}
\left\langle z^{*}(t) \circ P, y\right\rangle & =\left\langle z^{*}(t), P y\right\rangle \\
& =\left\langle z^{*}(t), x_{0}+P y\right\rangle-\left\langle z^{*}(t), x_{0}\right\rangle \\
& \leq f\left(t, x_{0}+P(y)\right)-f(t, 0)+\eta(t)-\left\langle z^{*}(t), x_{0}\right\rangle+\sigma_{N_{\mathrm{dom} I_{f}}(0)}\left(x_{0}+P(y)\right) \\
& \leq f\left(t, x_{0}+P(y)\right)-f(t, 0)+\eta(t)+\left|\left\langle z^{*}(t)-y^{*}(t), x_{0}\right\rangle\right|+\left|\left\langle y^{*}(t), x_{0}\right\rangle\right| \\
& \leq f\left(t, x_{0}+P(y)\right)-f(t, 0)+\eta(t)+\varepsilon(t)+\left|\left\langle y^{*}(t), x_{0}\right\rangle\right|,
\end{aligned}
$$

and the weak integrability of $z^{*}(\cdot) \circ P$ follows, as

$$
\begin{aligned}
\int_{T}\left|\left\langle z^{*}(t) \circ P, y\right\rangle\right| d \mu(t) \leq & \int_{T}\left|f\left(t, x_{0}+P(y)\right)\right| d \mu(t) \\
& +\int_{T}\left|f\left(t, x_{0}-P(y)\right)\right| d \mu(t)-I_{f}(0) \\
& +\int_{T}\left(\eta(t)+\varepsilon(t)+\left|\left\langle y^{*}(t), x_{0}\right\rangle\right|\right) d \mu(t)<+\infty .
\end{aligned}
$$

Moreover, (21) implies that $\int_{T} z^{*} \circ P d \mu$ is uniformly bounded on a neighborhood of zero, so that

$$
\int_{T} z^{*} \circ P d \mu \in X^{*}
$$

Finally, we have $z^{*}(t) \circ P=z^{*}(t)+z^{*}(t) \circ P-z^{*}(t)$,

$$
z^{*}(t) \in \partial_{\eta(t)} f_{t}(0)+N_{\operatorname{dom} I_{f}}(0) \text { and } z^{*}(t) \circ P-z^{*}(t) \in F^{\perp},
$$

and, consequently,

$$
z^{*}(t) \circ P \in \partial_{\eta(t)} f_{t}(0)+N_{\operatorname{dom} I_{f}}(0)+F^{\perp}=\partial_{\eta(t)} f_{t}(0)+N_{\operatorname{dom} I_{f}}(0)
$$

and (recall (20) $)$

$x^{*}-\int_{T} z^{*} \circ P d \mu=x^{*}-\int_{T} y^{*} d \mu+\int_{T}\left(y^{*}-z^{*}\right) d \mu-\int_{T}\left(z^{*} \circ P-z^{*}\right) d \mu \in V+V+F^{\perp}$.

So, observing that $F^{\perp} \subset \int_{T} N_{\operatorname{dom} I_{f}}(0) d \mu(t)$,

$$
\begin{aligned}
x^{*} & \in(w)-\int_{T}\left(\partial_{\eta(t)} f_{t}(0)+N_{\operatorname{dom} I_{f}}(0)\right) d \mu(t)+V+V+F^{\perp} \\
& \subset(w)-\int_{T}\left(\partial_{\eta(t)} f_{t}(0)+N_{\operatorname{dom} I_{f}}(0)\right) d \mu(t)+V+V .
\end{aligned}
$$

Hence, by intersecting over $V$ we get

$$
x^{*} \in \mathrm{cl}^{w^{*}}\left\{(w)-\int_{T}\left(\partial_{\eta(t)} f_{t}(x)+N_{\operatorname{dom} I_{f}}(x)\right) d \mu(t)\right\},
$$

which gives the desired inclusion due to the arbitrariness of the function $\eta$. 
Remark 7 If in Theorem 5. $X$ is a Banach space and, for a given point $\bar{x}$, there exists some sequence $\left(\eta_{n}\right)_{n} \subset L^{1}(T,(0,+\infty))$ such that

$$
\int_{T} \eta(t) d \mu(t) \rightarrow 0, \quad(w)-\int_{T} \partial_{\eta_{n}(t)} f_{t}(\bar{x}) d \mu(t) \neq \emptyset, \forall n \in \mathbb{N},
$$

then

$$
\partial I_{f}(\bar{x})=\bigcap_{n \in \mathbb{N}} \mathrm{cl}^{w^{*}}\left\{(w)-\int_{T} \partial_{\eta_{n}(t)} f_{t}(\bar{x}) d \mu(t)+N_{\operatorname{dom} I_{f}}(\bar{x})\right\} .
$$

Indeed, the inclusion " $\subseteq$ " is straightforward. Moreover, for each $n \in \mathbb{N}$ we have that (due to the fact that the integral of $\partial_{\eta_{n}(\cdot)} f .(\bar{x})$ and $N_{\operatorname{dom} I_{f}}(\bar{x})$ are nonempty)

$(w)-\int_{T}\left(\partial_{\eta(t)} f_{t}(x)+N_{\operatorname{dom} I_{f}}(x)\right) d \mu(t) \subseteq \mathrm{cl}^{w^{*}}\left\{(w)-\int_{T} \partial_{\eta_{n}(t)} f_{t}(\bar{x}) d \mu(t)+N_{\operatorname{dom} I_{f}}(\bar{x})\right\}$

(see e.g. [20, Proposition 5.6], [1, Proposition 8.6.2]). Then, by (18), the left-hand side is included in the right-hand side.

Corollary 5 Let $(T, \Sigma, \mu)$ be a finite-measure space and let $f: T \times \mathbb{R}^{n} \rightarrow \overline{\mathbb{R}}$ be a convex normal integrand. Then, for every $x \in \mathbb{R}^{n}$,

$$
\partial I_{f}(x)=\bigcap_{\eta>0} \operatorname{cl}\left\{\int_{T}\left(\partial_{\eta} f_{t}(x)+N_{\operatorname{dom} I_{f}}(x)\right) d \mu(t)\right\}
$$

Proof Since the measure is finite we have that the right-hand side of (23) is included in $\partial I_{f}(x)$. Moreover, since the constant positive functions belongs to $L^{1}(T,(0,+\infty))$, Theorem 5 implies that the subdifferential of $I_{f}$ at $x$ is included in the right-hand side of (23).

\section{Conclusions}

In this work, we presented general characterizations of the $\varepsilon$-subdifferential of the integral functional $I_{f}$, without any qualification conditions, when defined over a locally convex space (see Theorem 3 for the main result). We used a finitedimensional reduction approach since there is no theory about measurable selections and integration of multifunctions in non-separable locally convex space. We provided simplifications under qualification conditions of the nominal data (see Corollary 2).

Acknowledgements. We very much appreciate the insightful suggestions and helpful comments of the referees, which have contributed to improving the current revision of the manuscript. 


\section{References}

1. J.-P. Aubin and H. Frankowska. Set-valued analysis. Modern Birkhäuser Classics. Birkhäuser Boston, Inc., Boston, MA, 2009.

2. R. J. Aumann. Integrals of set-valued functions. J. Math. Anal. Appl., 12:1-12, 1965.

3. J. M. Borwein and A. S. Lewis. Convex analysis and nonlinear optimization, volume 3 of CMS Books in Mathematics/Ouvrages de Mathématiques de la SMC. Springer, New York, second edition, 2006. Theory and examples.

4. J. M. Borwein and J. D. Vanderwerff. Convex functions: constructions, characterizations and counterexamples, volume 109 of Encyclopedia of Mathematics and its Applications. Cambridge University Press, Cambridge, 2010.

5. J M. Borwein and L. Yao. Legendre-type integrands and convex integral functions. J. Convex Anal., 21(1):261-288, 2014.

6. R. I. Bot, S. M. Grad, and G. Wanka. New constraint qualification and conjugate duality for composed convex optimization problems. J. Optim. Theory Appl., 135(2):241-255, 2007.

7. N. Bourbaki. Éléments de mathématique. VIII. Première partie: Les structures fondamentales de l'analyse. Livre III: Topologie générale. Chapitre IX: Utilisation des nombres réels en topologie générale. Actualités Sci. Ind., no 1045. Hermann et Cie., Paris, 1948.

8. R. S. Burachik and V. Jeyakumar. A dual condition for the convex subdifferential sum formula with applications. J. Convex Anal., 12(2):279-290, 2005.

9. C. Castaing. Sur les multi-applications mesurables. Rev. Française Informat. Recherche Opérationnell, 1(1):91-126, 1967.

10. C. Castaing and M. Valadier. Convex analysis and measurable multifunctions. Lecture Notes in Mathematics, Vol. 580. Springer-Verlag, Berlin-New York, 1977.

11. C. Combari, M. Laghdir, and L. Thibault. On subdifferential calculus for convex functions defined on locally convex spaces. Ann. Sci. Math. Québec, 23(1):23-36, 1999.

12. R. Correa, A. Hantoute, and M. A. López. Towards supremum-sum subdifferential calculus free of qualification conditions. SIAM J. Optim., 26(4):2219-2234, 2016.

13. R. Correa, A. Hantoute, and M. A. López. Weaker conditions for subdifferential calculus of convex functions. J. Funct. Anal., 271(5):1177-1212, 2016.

14. R. Correa, A. Hantoute, and P. Pérez-Aros. Sequential and exact formulae for the subdifferential of nonconvex integral functionals. ArXiv e-prints, 2018. 2803.05521.

15. R. Correa, A. Hantoute, and P. Pérez-Aros. Characterizations of the subdifferential of convex integral functions under qualification conditions. Journal of Functional Analysis, 277(1):227 - 254, 2019.

16. J. Diestel and J. J. Uhl, Jr. Vector measures. American Mathematical Society, Providence, R.I., 1977. With a foreword by B. J. Pettis, Mathematical Surveys, No. 15.

17. A. Hantoute, M. A. López, and C. Zălinescu. Subdifferential calculus rules in convex analysis: a unifying approach via pointwise supremum functions. SIAM J. Optim., 19(2):863$882,2008$.

18. J.-B. Hiriart-Urruty and C. Lemaréchal. Fundamentals of convex analysis. Grundlehren Text Editions. Springer-Verlag, Berlin, 2001. Abridged version of it Convex analysis and minimization algorithms. I [Springer, Berlin, 1993; MR1261420 (95m:90001)] and it II [ibid.; MR1295240 (95m:90002)].

19. J.-B. Hiriart-Urruty, M. Moussaoui, A. Seeger, and M. Volle. Subdifferential calculus without qualification conditions, using approximate subdifferentials: a survey. Nonlinear Anal., 24(12):1727-1754, 1995.

20. S. Hu and N. S. Papageorgiou. Handbook of multivalued analysis. Vol. I, volume 419 of Mathematics and its Applications. Kluwer Academic Publishers, Dordrecht, 1997. Theory.

21. A. D. Ioffe. Survey of measurable selection theorems: Russian literature supplement. SIAM J. Control Optim., 16(5):728-732, 1978.

22. A. D. Ioffe. Three theorems on subdifferentiation of convex integral functionals. J. Convex Anal., 13(3-4):759-772, 2006.

23. A. D. Ioffe and V. L. Levin. Subdifferentials of convex functions. Trudy Moskov. Mat. Ob̌̌č., 26:3-73, 1972 .

24. A. D. Ioffe and V. M. Tikhomirov. Duality of convex functions and extremum problems. Russian Mathematical Surveys, 23(6):53, 1968.

25. P.-J. Laurent. Approximation et optimisation. Hermann, Paris, 1972.

26. V. L. Levin. Convex integral functionals and the theory of lifting. Russian Mathematical Surveys, 30(2):119, 1975. 
27. O. Lopez and L. Thibault. Sequential formula for subdifferential of integral sum of convex functions. J. Nonlinear Convex Anal., 9(2):295-308, 2008.

28. B. S. Mordukhovich and N. Sagara. Subdifferentials of nonconvex integral functionals in banach spaces with applications to stochastic dynamic programming. J. Convex Anal., 25(2):643-673, 2018.

29. J.-J. Moreau. Fonctionnelles convexes. Séminaire Jean Leray, (2):1-108, 1967.

30. P. Pérez-Aros. Formulae for the conjugate and the subdifferential of the supremum function. Journal of Optimization Theory and Applications, 180(2):397-427, 2019.

31. P. Pérez-Aros. Subdifferential formulae for the supremum of an arbitrary family of functions. SIAM J. Optim., 29(2):1714-1743, 2019.

32. R. T. Rockafellar. Integrals which are convex functionals. Pacific J. Math., 24:525-539, 1968.

33. R. T. Rockafellar. Convex integral functionals and duality. In Contributions to nonlinear functional analysis (Proc. Sympos., Math. Res. Center, Univ. Wisconsin, Madison, Wis., 1971), pages 215-236. Academic Press, New York, 1971.

34. R. T. Rockafellar. Integrals which are convex functionals. II. Pacific J. Math., 39:439-469, 1971.

35. R. T. Rockafellar. Integral functionals, normal integrands and measurable selections. pages 157-207. Lecture Notes in Math., Vol. 543, 1976.

36. R. T. Rockafellar. Convex analysis. Princeton Landmarks in Mathematics. Princeton University Press, Princeton, NJ, 1997. Reprint of the 1970 original, Princeton Paperbacks.

37. R. T. Rockafellar and R. J.-B. Wets. Variational analysis, volume 317 of Grundlehren der Mathematischen Wissenschaften [Fundamental Principles of Mathematical Sciences]. Springer-Verlag, Berlin, 1998.

38. L. Schwartz. Radon measures on arbitrary topological spaces and cylindrical measures. Published for the Tata Institute of Fundamental Research, Bombay by Oxford University Press, London, 1973. Tata Institute of Fundamental Research Studies in Mathematics, No. 6.

39. C. Vallée and C. Zălinescu. Series of convex functions: subdifferential, conjugate and applications to entropy minimization. J. Convex Anal., 23(4):1137-1160, 2016.

40. D. H. Wagner. Survey of measurable selection theorems. SIAM J. Control Optimization, 15(5):859-903, 1977.

41. D. H. Wagner. Survey of measurable selection theorems: an update. In Measure theory, Oberwolfach 1979 (Proc. Conf., Oberwolfach, 1979), volume 794 of Lecture Notes in Math., pages 176-219. Springer, Berlin-New York, 1980.

42. C. Zălinescu. Convex analysis in general vector spaces. World Scientific Publishing Co., Inc., River Edge, NJ, 2002. 